\title{
Olivine-dominated asteroids: Mineralogy and origin
}

Juan A. Sanchez ${ }^{\mathrm{a}, 1, *}$, Vishnu Reddy ${ }^{\mathrm{b}, 1}$, Michael S. Kelley ${ }^{\mathrm{c}, 1,2}$, Edward A. Cloutis ${ }^{\mathrm{d}}$, William F. Bottke $^{\mathrm{e}}$, David Nesvornýe ${ }^{\mathrm{e}}$, Michael P. Lucas ${ }^{\mathrm{f}}$, Paul S. Hardersen ${ }^{\mathrm{g}, 1}$, Michael J. Gaffeyg,1, Paul A. Abell ${ }^{\mathrm{h}, 1}$, Lucille Le Corre ${ }^{\mathrm{b}}$

\author{
${ }^{a}$ Max Planck Institut für Sonnensystemforschung, Katlenburg-Lindau, Germany \\ ${ }^{b}$ Planetary Science Institute, 1700 East Fort Lowell Road, Tucson, Arizona 85719, USA \\ ${ }^{c}$ Department of Geology and Geography, Georgia Southern University, Statesboro, USA \\ ${ }^{d}$ Department of Geography, University of Winnipeg, Winnipeg, Manitoba, Canada \\ ${ }^{e}$ Southwest Research Institute and NASA Lunar Science Institute, Boulder, USA \\ ${ }^{f}$ Department of Earth and Planetary Sciences, University of Tennessee, USA \\ ${ }^{g}$ Department of Space Studies, University of North Dakota, Grand Forks, USA \\ ${ }^{h}$ NASA Johnson Space Center, Houston, Texas, USA
}

\begin{abstract}
Olivine-dominated asteroids are a rare type of objects formed either in nebular processes or through magmatic differentiation. The analysis of meteorite samples suggest that at least 100 parent bodies in the main belt experienced partial or complete melting and differentiation before being disrupted. However, only a few olivine-dominated asteroids, representative of the mantle of disrupted differentiated bodies, are known to exist. Due to the paucity of these objects in the main belt their origin and evolution have been a matter of great debate over the years. In this work we present a detailed mineralogical analysis of twelve olivine-dominated asteroids. We have obtained near-infrared (NIR) spectra (0.7 to $2.4 \mu \mathrm{m})$ of asteroids (246) Asporina, (289) Nenetta, (446) Aeternitas, (863) Benkoela, (4125) Lew Allen and (4490) Bamberry. Observations were conducted with the Infrared Telescope Facility (IRTF) on Mauna Kea, Hawai'i. This sample was complemented with spectra of six other olivine-dominated asteroids including (354) Eleonora, (984) Gretia, (1951) Lick, (2501) Lohja, (3819) Robinson and (5261) Eureka obtained by previous workers. Within our sample we distinguish two classes, one that we call monomineralic-olivine asteroids, which are those whose spectra only exhibit the $1 \mu \mathrm{m}$ feature, and another referred to as olivine-rich asteroids, whose spectra exhibit the $1 \mu \mathrm{m}$ feature and a weak (Band II depth 4\%) $2 \mu \mathrm{m}$ feature. For the monomineralic-olivine asteroids the olivine chemistry was found to range from $\sim \mathrm{Fo}_{49}$ to $\mathrm{Fo}_{70}$, consistent with the values measured for brachinites and $\mathrm{R}$ chondrites. In the case of the olivine-rich asteroids we determined their olivine and low-Ca pyroxene abundance using a new set of spectral calibrations derived from the analysis of $\mathrm{R}$ chondrites spectra. We found that the olivine abundance for these asteroids varies from 0.68 to 0.93 , while the fraction of low-Ca pyroxene to total pyroxene ranges from 0.6 to 0.9. A search for dynamical connections between the olivine-dominated asteroids and asteroid families found no genetic link (of the type core-mantel-crust) between these objects.
\end{abstract}

Keywords:

${ }^{*}$ Corresponding author at: Max Planck Institut für Sonnensystemforschung, Max Planck Str.2, 37191 Katlenburg-Lindau, Germany.

Email address: sanchez@mps.mpg.de (Juan A. Sanchez)

${ }^{1}$ Visiting Astronomer at the Infrared Telescope Facility, which is operated by the University of Hawaii under Cooperative Agreement No. NNX-08AE38A with the National Aeronautics and Space Administration, Science Mission Directorate, Planetary Astronomy Program.

${ }^{2}$ Planetary Science Division, Science Mission Directorate, NASA Headquarters, Washington, DC 20546, USA. 


\section{Introduction}

A-type asteroids are a unique class of objects that were initially distinguished from the R-type asteroids (the group into which they'd previously been classified) based on broadband spectrophotometry by Veeder et al. (1983) and were later re-classified based on Eight Color Asteroid Survey (ECAS) data $(0.3$ to $1.1 \mu \mathrm{m})$ by Tholen (1984). Asteroids of this taxonomic class have moderately high albedos, extremely reddish slopes shortward of $0.7 \mu \mathrm{m}$, and a strong absorption feature centered at $\sim 1.05 \mu \mathrm{m}$ (Tholen and Barucci, 1989). Subsequent near-infrared (NIR) spectra have shown that in these original A-type asteroids a $\sim 2 \mu \mathrm{m}$ feature is absent or very weak, consistent with a silicate component of nearly monomineralic olivine on the surface of these bodies.

The discovery of olivine-dominated asteroids is of considerable interest regarding the accretion and geochemical evolution of primitive bodies. Olivine-dominated objects are expected to form either through magmatic differentiation, being the major constituent of the mantles of most differentiated bodies (Burbine et al., 1996), or through nebular processes which can produce olivine-dominated objects like the R-chondrite parent body (Schulze et al., 1994). The presence of olivine-dominated asteroids suggests that at least some objects in the asteroid belt underwent complete or near-complete melting that led to the differentiation of their interiors. Another interesting aspect is that in order for the mantle to be exposed, the parent body must be fragmented or its deep interior exposed by large impacts. Based on meteorites in terrestrial collections, it is estimated that at least $\sim 100$ meteorite parent bodies should have existed in the asteroid belt that underwent partial or complete melting and differentiation before disruption and fragmentation (Keil, 2000). However, even assuming all A-type asteroids are olivinedominated, only a handful of objects from the mantles of differentiated and disrupted parent bodies were discovered during the taxonomic surveys. This is described as the "missing mantle" problem because the corresponding mantle components of the iron cores (as represented by iron meteorites) are missing (Chapman, 1986; Bell et al., 1989; Burbine et al., 1996). More recent work on M-type asteroids by Hardersen et al. (2011) indicates that a subset of that population (766 Moguntia, 798 Ruth and 1210 Morosovia) shows a significant olivine component in the surface assemblage. It is unclear, at this point, if the olivine seen on these M-type asteroids are pieces of mantle remaining on an iron-rich core or formed via nebular processes.

More recent surveys like the Small Main-Belt Asteroid Spectroscopic Survey (SMASS) (Xu et al., 1995), and SMASS II (Bus and Binzel, 2002a b) have expanded the number of members within each taxonomic class based on visible spectroscopy. Twelve new A-type asteroids were added to the original five from Tholen (1984). Burbine and Binzel (2002) observed 10 A-type asteroids at near-infrared wavelengths, four from Tholen (1984) and six from Bus and Binzel (2002a, b). They subsequently divided the A-type asteroids into two groups based on the strength of the $1 \mu \mathrm{m}$ feature. Because the Burbine and Binzel (2002) data do not extend beyond $1.65 \mu \mathrm{m}$, the possibility of a $2 \mu \mathrm{m}$ feature due to pyroxene cannot be ruled out. It is important to note that A-type asteroids under the Bus and Binzel (2002a, b) taxonomic system are not the same as those under the original Tholen system. Some A-types in the SMASS II taxonomic system contain up to $\sim 20 \%$ pyroxene, as indicated by the presence of a $2 \mu \mathrm{m}$ feature in NIR data, e.g., (4142) Dersu-Uzala (Binzel et al., 2004), and are similar to S-I/S-II asteroids in the Gaffey S-asteroid subtypes (Gaffey et al., 1993).

A comprehensive summary of all previous work on A-type asteroids is published in Sunshine et al. (2007). This study was based on the work of Sunshine and Pieters (1998), and included VIS-NIR spectra of nine olivine-dominated asteroids. Of these nine objects, four were 
analyzed using the Modified Gaussian Model (MGM) (Sunshine et al., 1990) in order to derive their olivine compositions. Those four objects that included (1951) Lick, (289) Nenetta, (246) Asporina, and (354) Eleonora were characterized by the lack of a detectable $2 \mu \mathrm{m}$ feature. The other five asteroids; (446) Aeternitas, (863) Benkoela, (984) Gretia, (2501) Lohja, and (3819) Robinson, whose spectra have a detectable $2 \mu \mathrm{m}$ feature, were not analyzed due to the difficulties inherent to the modeling of olivine-pyroxene mixtures (Sunshine et al., 2007).

In the present work we analyze VIS-NIR spectra of twelve olivine-dominated asteroids, six observed by our group and six obtained from previous studies. Because taxonomic classification can be ambiguous depending on the system used we will refer to these objects as S(I)-types, which is the designation introduced by Gaffey et al. (1993) that includes objects where olivine is the major silicate phase present. We further distinguish two classes within our sample: one class that will be called monomineralic-olivine asteroids, which are those whose spectra exhibit the $1 \mu \mathrm{m}$ feature and no detectable $2 \mu \mathrm{m}$ feature, and another class that will be called olivine-rich asteroids, whose spectra exhibit the $1 \mu \mathrm{m}$ feature and a weak $2 \mu \mathrm{m}$ feature.

The approach used in the present study differs from previous work (e.g., Sunshine and Pieters, 1998; Sunshine et al., 2007) in that olivine compositions are determined from the measured Band I centers, along with a spectral calibration derived from laboratory measurements. Furthermore, in the case of the olivine-rich asteroids, the olivine-pyroxene abundance ratio $(\mathrm{ol} /(\mathrm{ol}+\mathrm{px}))$ and the ratio of low-Ca pyroxene (LCP) to total pyroxene $(\mathrm{LCP} /(\mathrm{LCP}+\mathrm{HCP}))$ are determined using a set of equations derived from the analysis of meteorite samples. Here we define low-Ca pyroxenes (LCP) as pyroxenes with $<25 \%$ iron and include pigeonite and orthopyroxene, and high-Ca pyroxenes (HCP) as those with $>25 \%$ iron, including augite-diopside-hedenbergite.

In addition to the mineralogical analysis we also search for dynamical connections between the studied objects and asteroid families. Using this information we finally discuss possible formation scenarios for the olivine-dominated asteroids.

\section{Observations and data reduction}

Observations were carried out with the NASA IRTF on Mauna Kea, Hawai'i. NIR spectra ( 0.7-2.5 $\mu \mathrm{m}$ ) were obtained with the SpeX instrument (Rayner et al., 2003) in its low resolution (R 150) prism mode with a 0.8 " slit width. A typical observing sequence consists of spectra taken in pairs (A-beam and B-beam) by nodding the telescope. Nodding enables the subtraction of the sky background from the object during the data reduction process. Depending on the magnitude of the asteroid, 10-20 spectra are taken per asteroid with a maximum integration of 120 seconds due to saturation from the background sky. In order to correct for telluric water vapor features and to obtain relative reflectance values, local standard and solar analog stars were also observed. For each night, flat fields and arc line spectra were acquired. Data reduction was carried out with Spextool (Cushing et al., 2004). Detailed descriptions of observation and data reduction protocols are presented in Reddy (2009) and Reddy et al. (2011a, 2012a). NIR spectra are normalized to unity at $1.5 \mu \mathrm{m}$. Table 1 lists observational circumstances for the observed asteroids.

In order to extend our study we also analyzed data from Sunshine et al. (2007), de León et al. (2010), and the MIT-UH-IRTF Joint Campaign for NEO Spectral Reconnaissance (NEOSR). NIR spectra were combined with VIS spectra in order to increase the wavelength coverage. VIS spectra were obtained from the SMASS II (Bus and Binzel, 2002a, b), and the Small Solar System Objects Spectroscopic Survey $\left(\mathrm{S}^{3} \mathrm{OS}^{2}\right)$ (Lazzaro et al., 2004). VIS-NIR spectra of the studied asteroids are shown in Figs. 1 and 2 . 
Table 1: Observational circumstances. The columns in this table are: object number and designation, UT date, number of exposures, phase angle $(\alpha)$, V-magnitude at the time of observation, heliocentric distance (r), air mass and solar analog used. The integration time with IRTF/SpeX was $120 \mathrm{~s}$.

\begin{tabular}{|c|c|c|c|c|c|c|c|}
\hline Object & UT date & $\operatorname{Exp}$ & $\alpha\left(^{\circ}\right)$ & mag. $(\mathrm{V})$ & $\mathrm{r}(\mathrm{AU})$ & Air mass & Solar analog \\
\hline 246 Asporina & 20-Jan-2004 & 10 & 5.5 & 15.0 & 2.88 & 1.16 & Hyades 64 \\
289 Nenetta & 17-Feb-2003 & 16 & 15.0 & 15.4 & 3.46 & 1.16 & Hyades 64 \\
446 Aeternitas & 20-Jan-2004 & 10 & 13.7 & 12.4 & 3.13 & 1.00 & Hyades 64 \\
863 Benkoela & 17-Nov-2002 & 6 & 17.9 & 14.8 & 3.20 & 1.02 & Hyades 64 \\
4125 Lew Allen & 15-Aug-2003 & 12 & 21.2 & 15.0 & 1.70 & 1.00 & BS5996 \\
4490 Bamberry & 17-Feb-2003 & 18 & 24.2 & 15.7 & 1.88 & 1.04 & Hyades 64 \\
\hline
\end{tabular}
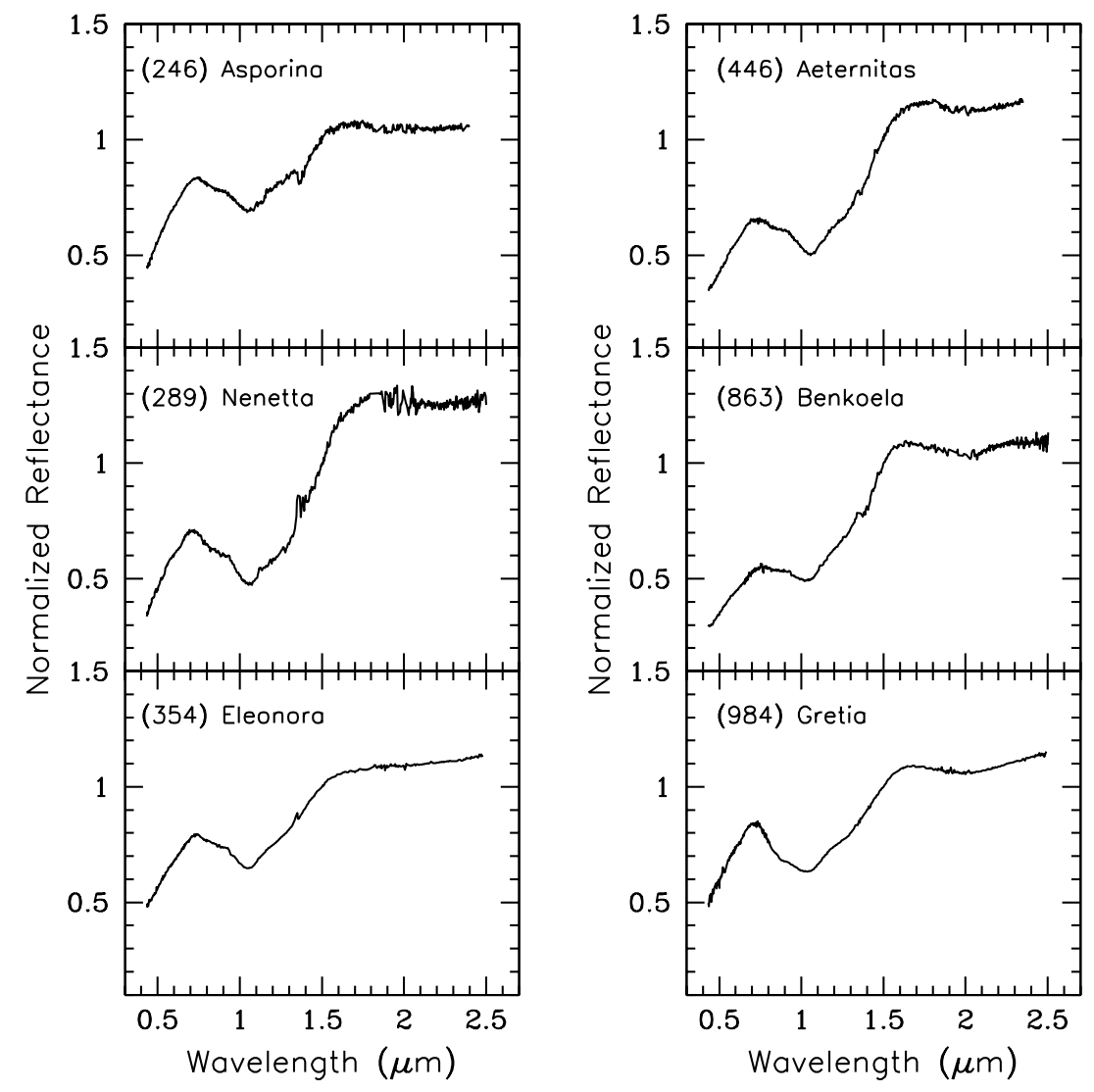

Figure 1: VIS-NIR spectra of asteroids analyzed in this study. All spectra are normalized to unity at $1.5 \mu \mathrm{m}$. The noise seen in the spectrum of (289) Nenetta longwards of $1.9 \mu \mathrm{m}$ is due to incompletely corrected telluric bands. The NIR spectrum of (354) Eleonora was obtained from the NEOSR survey (http://smass.mit.edu/minus.html). The NIR spectrum of (984) Gretia was obtained from Sunshine et al. (2007). VIS spectra of all asteroids were obtained from the SMASS II survey (Bus and Binzel, 2002a, b). 

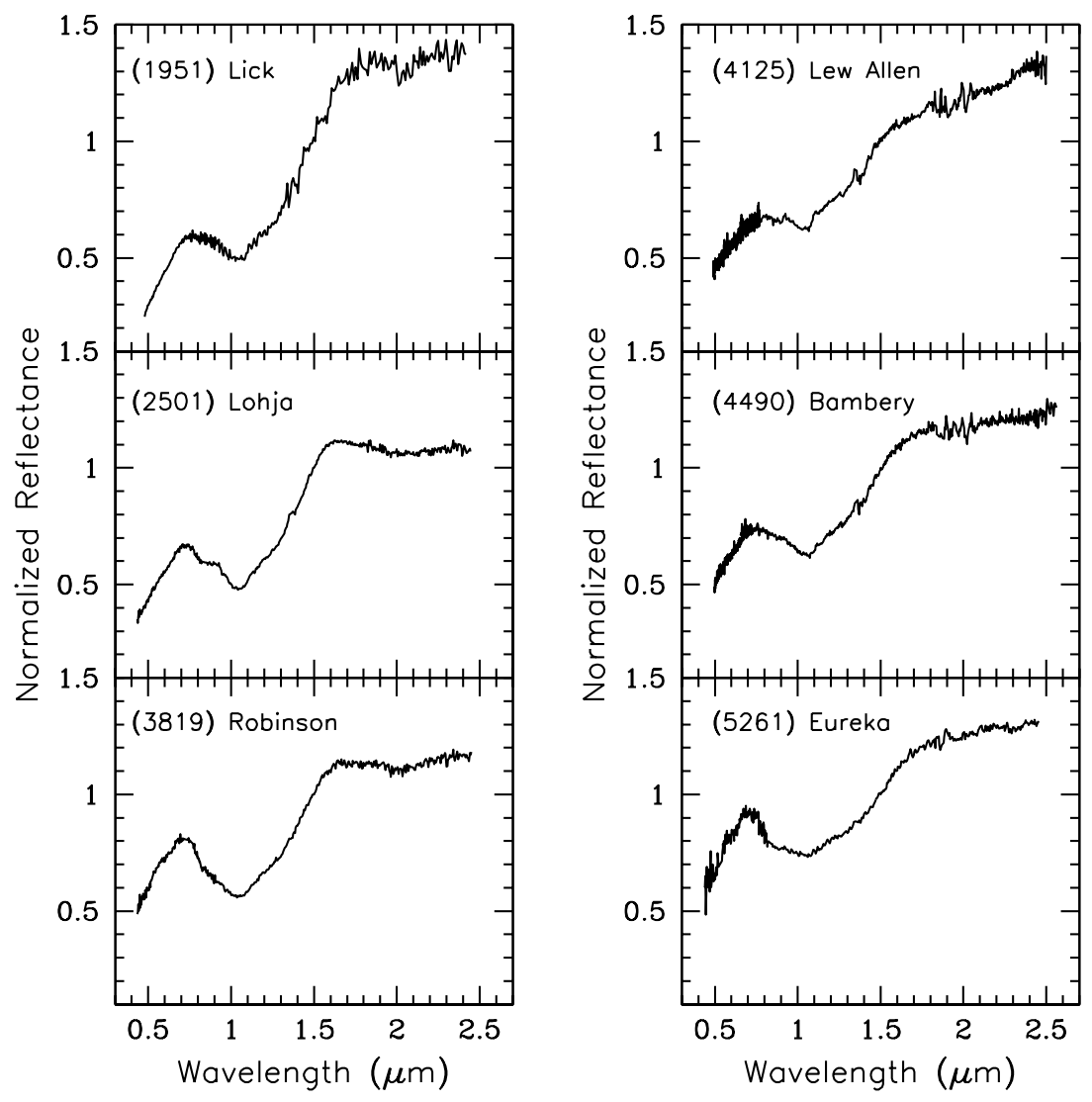

Figure 2: VIS-NIR spectra of asteroids analyzed in this study. All spectra are normalized to unity at $1.5 \mu \mathrm{m}$. The noise seen in the spectra of (1951) Lick, (4125) Lew Allen and (4490) Bambery longwards of $1.9 \mu \mathrm{m}$ is due to incompletely corrected telluric bands. The VIS-NIR spectrum of (1951) Lick was obtained from de León et al. (2010), the NIR spectrum of (2501) Lohja was obtained from Sunshine et al. (2007), NIR spectra of (3819) Robinson and (5261) Eureka were obtained from the NEOSR survey (http://smass.mit.edu/minus.html). VIS spectra of asteroids (2501) Lohja. (3819) Robinson, and (5261) Eureka were obtained from the SMASS II survey (Bus and Binzel, 2002a, b). VIS spectra of asteroids (4125) Lew Allen and (4490) Bambery were obtained from the $S^{3} \mathrm{OS}^{2}$ survey (Lazzaro et al., 2004). 


\section{Results}

\subsection{Monomineralic-olivine asteroids}

Asteroids in our sample that fall into the class of monomineralic-olivines are: (246) Asporina, (289) Nenetta, (354) Eleonora, (1951) Lick, (4125) Lew Allen, (4490) Bambery, and (5261) Eureka. The spectra of these asteroids lack detectable $2 \mu \mathrm{m}$ feature (Band II depth $<1 \%$ ), and the combined VIS-NIR spectra typically overlap at $\sim 0.7 \mu \mathrm{m}$. For each spectrum the Band I center and Band I depth are calculated after dividing out the linear continuum (a straight line tangent to the reflectance maxima) and fitting a polynomial over the bottom third of the band. A detailed explanation about the procedure used to measure the band parameters and their uncertainties can be found in Sanchez et al. (2012, 2013). The band parameters with their corresponding errors are presented in Table 2

Table 2: Spectral band parameters for the monomineralic-olivine asteroids. The columns in this table correspond to: object number and designation, Band I center (BIC \pm 0.005$)$, temperature corrected Band I center $(\Delta \mathrm{BIC} \pm 0.005)$, olivine composition $(\mathrm{Fo} \pm 5 \%)$ and its temperature corrected value $(\Delta \mathrm{Fo} \pm 5 \%)$, Band I depth $\left(\mathrm{BI}_{\mathrm{depth}} \pm 0.3\right)$, geometric albedo $\left(p_{v}\right)$, the beaming parameter $(\eta)$ and the average surface temperature $(\mathrm{T})$. Temperature values were calculated in the same way as Burbine et al. (2009). The infrared emissivity, $\varepsilon$ is assumed to be 0.9 , albedo and $\eta$ values of asteroids (246) Asporina, (354) Eleonora, and (4125) Lew Allen are obtained from Masiero et al. (2011). Albedo values for asteroids (289) Nenetta, (1951) Lick, and (4490) Bambery are obtained from Tedesco et al. (2004). A slope parameter $\mathrm{G}=0.15$ has been adopted for all the asteroids with the exception of (354) Eleonora, which has $\mathrm{G}=0.37$ (Masiero et al., 2011). If the albedo and $\eta$ of the asteroid are unknown a value of $p_{v}=0.2$ and $\eta=1$ is assumed.

\begin{tabular}{|c|c|c|c|c|c|c|c|c|}
\hline Object & $\mathrm{BIC}(\mu \mathrm{m})$ & $\Delta \mathrm{BIC}(\mu \mathrm{m})$ & $\mathrm{Fo}(\mathrm{mol} \%)$ & $\Delta \mathrm{Fo}(\mathrm{mol} \%)$ & $\mathrm{BI}_{\text {depth }}(\%)$ & $p_{v}$ & $\eta$ \\
\hline 246 Asporina & 1.065 & 1.070 & 66.3 & 56.5 & 24.00 & $0.2069 \pm 0.0294$ & $1.118 \pm 0.018$ & 160.3 \\
289 Nenetta & 1.069 & 1.074 & 58.5 & 48.8 & 48.08 & $0.2438 \pm 0.042$ & - \\
354 Eleonora & 1.060 & 1.065 & 76.0 & 66.3 & 27.04 & $0.1732 \pm 0.0324$ & $1.071 \pm 0.084$ & 169.8 \\
1951 Lick & 1.061 & 1.063 & 74.1 & 70.2 & 38.35 & $0.0895 \pm 0.020$ & - \\
4125 Lew Allen & 1.062 & 1.065 & 72.1 & 66.3 & 23.56 & $0.1462 \pm 0.0358$ & $1.249 \pm 0.033$ & 2047.5 \\
4490 Bambery & 1.066 & 1.070 & 64.3 & 56.5 & 29.11 & $0.2156 \pm 0.024$ & - & 204.0 \\
5261 Eureka & 1.071 & 1.074 & 54.6 & 48.8 & 27.70 & - & - \\
\hline
\end{tabular}

The primary diagnostic feature in the spectrum of olivine assemblages is composed of three overlapping features and is centered near $\sim 1 \mu \mathrm{m}$. This composite feature is attributed to electronic transitions of $\mathrm{Fe}^{2+}$ occupying both the M1 and M2 crystallographic sites (Burns, 1993). Using NIR diffuse spectral reflectance, King and Ridlev (1987) investigated apparent wavelength shifts as a function of mineral chemistry in the $\mathrm{Fe} / \mathrm{Mg}$ olivine series from forsterite $\mathrm{Fo}_{11}$ to $\mathrm{Fo}_{91}$. They found that the composite $1 \mu \mathrm{m}$ feature in olivine moves to longer wavelengths as the $\mathrm{Fe}^{2+}$ content increases.

Reddy et al. (2011b) improved the olivine calibration developed by King and Ridley (1987) by adding more $\mathrm{Mg}$-rich olivine (Fo85-93) samples. Reflectance spectra of these additional samples were obtained at the University of Winnipeg HOSERLab. This calibration plot is shown in Figure 4, where the solid line represents a linear fit to the data used by Reddy et al. $(2011 b)$. The equation that describes the linear fit is given by

$$
F o=-1946.6 \times(B I C)+2139.4
$$

Using this equation, along with the measured Band I centers (BIC), we estimated the molar $\%$ of forsterite (Fo) for the monomineralic-olivine asteroids. The root mean square error between the molar content of forsterite determined using Eq. (1) and the laboratory measurements is 5\%, 


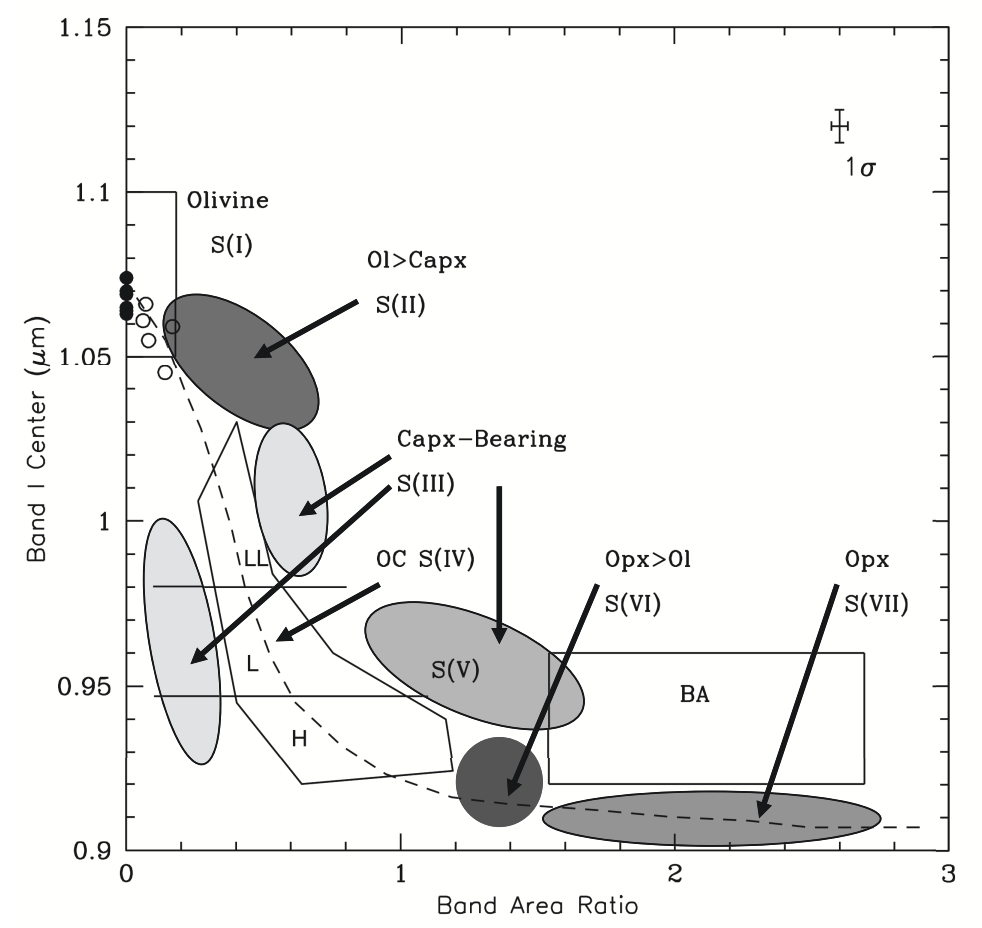

Figure 3: Plot of the Band I center versus BAR for the monomineralic-olivine (filled circles) and olivinerich (open circles) asteroids studied in the present work. The average 1- $\sigma$ error bars are shown in the upper right corner. The rectangular zone corresponding to the S(I)-type asteroids encompasses monomineralic olivine assemblages (Gaffey et al., 1993). The rectangular region (BA) includes the pyroxenedominated basaltic achondrite assemblages. The polygonal region, corresponding to the S(IV) subgroup, represents the mafic silicate components of ordinary chondrites (OC). The dashed curve indicates the location of the olivine-orthopyroxene mixing line (Cloutis et al., 1986). The horizontal lines represent the approximate boundaries for ordinary chondrites found by Dunn et al. (2010).

however due to the band center uncertainty the percentage of forsterite can only be estimated with a precision of $\pm 10-15 \%$.

The laboratory measurements used to develop Eq. (1) were obtained at room temperature $(300 \mathrm{~K})$, however the surface temperature of asteroids is typically much lower. The effects on spectral parameters of mafic minerals due to temperature have been investigated by several authors (e.g., Singer and Roush, 1985; Schade and Wäsch, 1999; Moroz et al., 2000; Hinrichs and Lucey, 2002; Reddy et al., 2012b; Sanchez et al., 2012). These effects are seen as broadening or narrowing of the absorption features and shifting of the band centers. In order to determine whether this temperature difference could affect the Band I centers measured from asteroid spectra, we have reanalyzed spectra of olivine ( $\mathrm{F}_{86}$ ) from Hinrichs and Lucev (2002) acquired in the temperature range between 80 and $400 \mathrm{~K}$, with a temperature resolution of 20 $\mathrm{K}$. Band I centers were measured using the same procedure applied to the asteroid spectra and then plotted as a function of temperature. We found that the Band I center shifts to longer wavelengths as the temperature increases. This shift in Band I center can be described as

$$
B I C(\mu m)=\left(1.18 \times 10^{-7}\right) T^{2}-\left(2.15 \times 10^{-5}\right) T+1.05
$$

From this equation we derived a wavelength correction with respect to room temperature $(300 \mathrm{~K})$ for the Band I center 


$$
\Delta B I C(\mu m)=-\left(1.18 \times 10^{-7}\right) T^{2}+\left(2.15 \times 10^{-5}\right) T+0.004
$$

This correction must be added to the calculated Band I center of each asteroid. The average surface temperature of the asteroids was calculated in the same way as Burbine et al. (2009). Thus, after correcting the Band I centers, Eq. (1) was used to calculate the olivine composition of the asteroids. The temperature corrected Band I centers, olivine compositions (with and without temperature corrections) and the average surface temperature of the asteroids are presented in Table 2, The temperature corrected Band I centers of the monomineralic-olivine asteroids are depicted in Fig. 3 as filled circles.

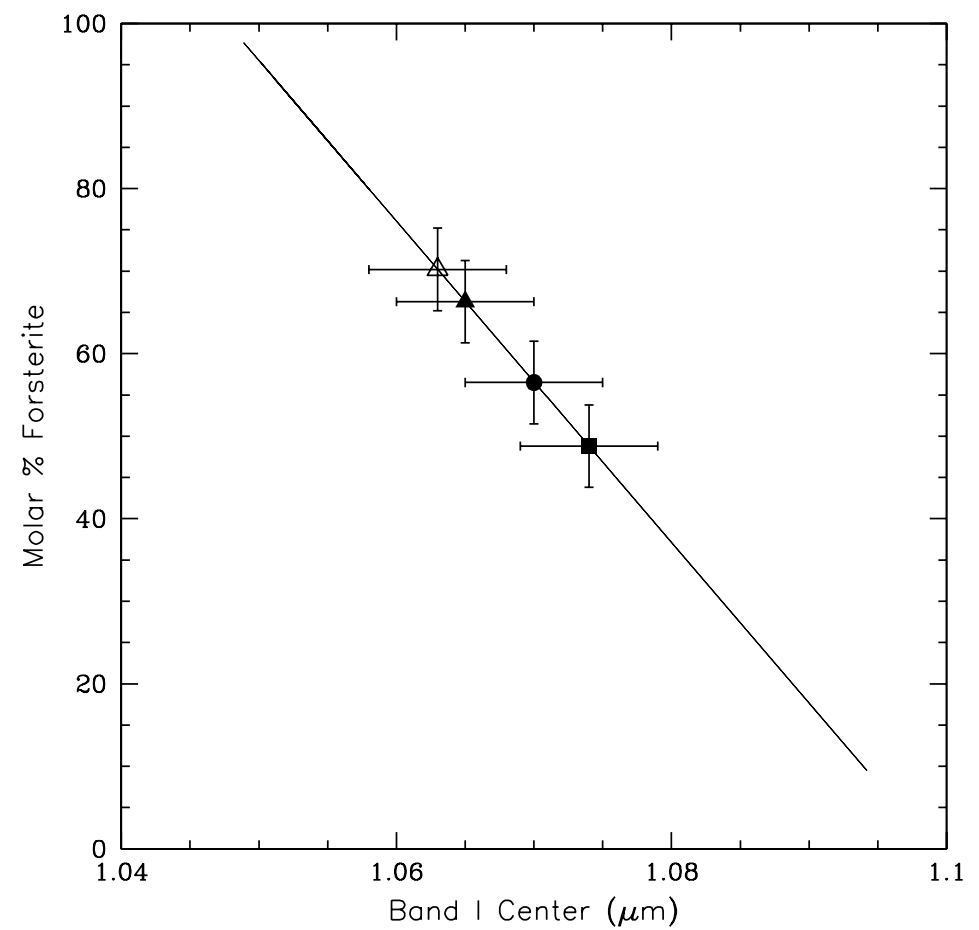

Figure 4: Improved olivine calibration plot developed by Reddy et al. (2011b). The linear relationship between the Band I center and forsterite content is represented as a solid line. The root mean square error between the molar content of forsterite determined using Eq. (1) and the laboratory measurements is $5 \%$. Also shown the calculated forsterite content for (246) Asporina and (4490) Bambery (both depicted as a filled circle), (289) Nenetta and (5261) Eureka (both depicted as a filled square), (354) Eleonora and (4125) Lew Allen (both depicted as a filled triangle), and (1951) Lick (open triangle).

\subsubsection{Mineralogical analysis}

Cruikshank and Hartmann (1984) were the first to obtain spectrophotometric observations in the NIR of (246) Asporina using the NASA IRTF, which led to the identification of nearly monomineralic-olivine on an asteroid surface. Using spectral curve matching techniques, the authors suggested that Asporina's spectrum best matched lab samples of olivine that were coarse grains mixed with "substantial fraction of grains $<45 \mu \mathrm{m}$ in size" (Cruikshank and Hartmann, 1984). Their best match for (246) Asporina was an olivine assemblage with mineralogy of $\mathrm{Fo}_{60-90}$. More recently, Sunshine et al. (2007) estimated the composition of (246) Asporina to be $\mathrm{Fo}_{63}$. The spectrum of (246) Asporina shown in Figure 1 is dominated by a deep complex feature (band depth $24 \%$ ) at $\sim 1 \mu \mathrm{m}$, and the derived temperature-corrected band center for this 
feature is $1.07 \mu \mathrm{m}$. The presence of this complex $1 \mu \mathrm{m}$ feature, composed of three overlapping narrower features, is a strong indication of the presence of olivine (Burns, 1993) with no high(Type B CPX) or low-Ca pyroxene due to the lack of $2 \mu \mathrm{m}$ feature. Using the calculated Band I center, and based on the olivine calibration by Reddy et al. (2011b) (Eq. 1) we found that Asporina has an estimated forsterite abundance of $\mathrm{FO}_{56.5 \pm 5}$. This value, depicted in Fig. 4 as a filled circle, is a little lower than the forsterite value reported for Asporina $\left(\mathrm{Fo}_{63}\right)$ by Sunshine et al. (2007).

After determining the olivine composition of Asporina the next step is to identify a possible meteorite analogue. S(I)-type asteroids have been traditionally interpreted as pieces of asteroid mantles based on the $1 \mu \mathrm{m}$ olivine spectral feature. While pallasites are often invoked as possible analogues, they are typically equated to core-mantle boundaries, and would therefore represent very deep mantle samples. Other olivine-rich meteorites that could be potential analogues for $\mathrm{S}(\mathrm{I})$ asteroids include the R-chondrites, brachinites, and ureilites. Representative spectra of these meteorites are shown in Fig. 5, for comparison we also included the spectrum of dunite NWA 2968 from Vesta measured at the University of Winnipeg HOSERLab for this study. This meteorite shows $\mathrm{FeO} / \mathrm{MnO}$ ratios and oxygen-isotopic compositions consistent with howardites, eucrites and diogenites (HED) meteorites, and therefore it is considered to be a fragment of Vesta's mantle.

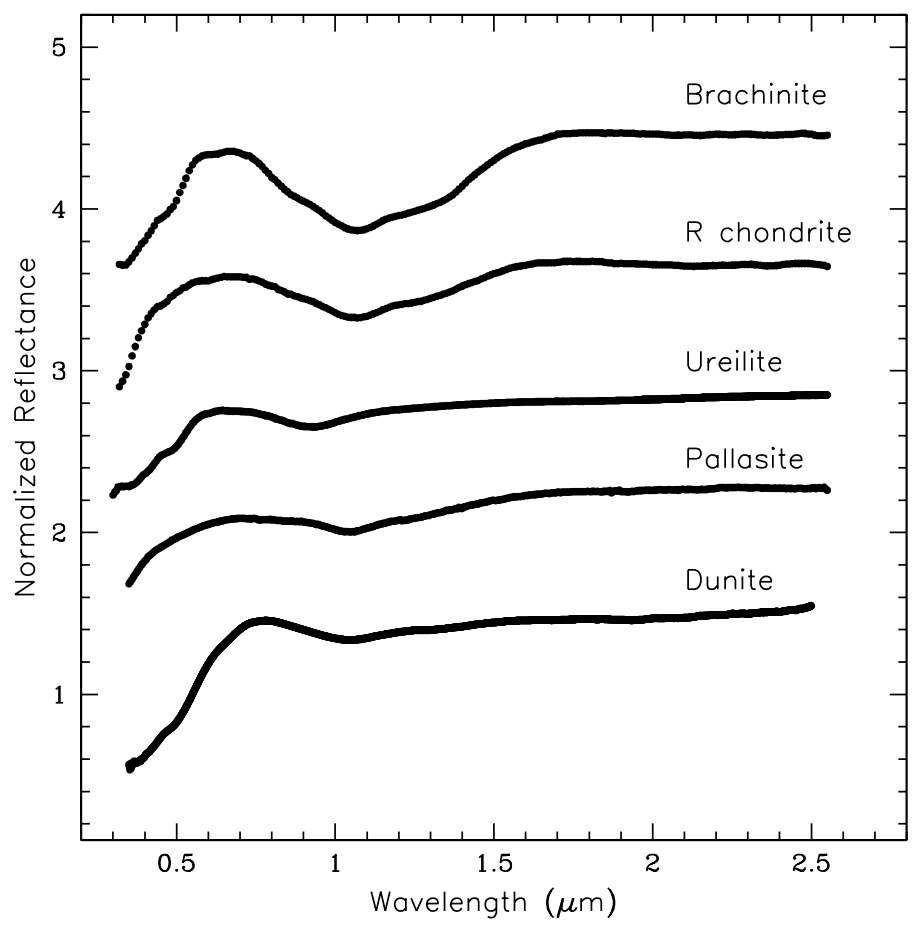

Figure 5: VIS-NIR spectra of olivine-dominated meteorites obtained from RELAB. All spectra are normalized to unity at $1.5 \mu \mathrm{m}$ and have been offset for clarity. From the bottom to the top: Dunite NWA 2968 from Vesta (measured at the University of Winnipeg HOSERLab), pallasite (Marjalahti, RELAB ID:MS-CMP-005-X), ureilite (Y-791538, RELAB ID:MP-TXH-101), R chondrite (Rumuruti, RELAB ID:MT-TJM-013), and Brachinite (EET99402, RELAB ID:TB-TJM-058).

Pallasites consist of metal and silicate (olivine) in roughly equal amounts with troilite as a minor phase (Mittlefehldt et al., 1998). The three types of pallasites (main group, Eagle station, and pyroxene-pallasite) differ from each other based on mineralogy, composition, and 
O-isotopes of the silicate and metal components. All three pallasite groups have olivine with Fo $_{80-90}$ (Mittlefehldt et al., 1998; Rubin, 1997), which is much higher than Asporina. Work by Cloutis et al. (1990) has shown that silicate spectral features are severely suppressed with the addition of metal, and significant metal (>50\%) can cause reddening of spectral slope. An example of a pallasite spectrum is depicted in Fig. 5 that shows the VIS-NIR spectrum of pallasite Marjalahti obtained from the RELAB database (Pieters and Hiroi, 2004). Based on the mismatch in olivine chemistry, and spectral parameters (band depth suppression and red spectral slope) we conclude that pallasites are not good meteorite analogues for (246) Asporina.

Ureilites are composed mostly of mafic silicates like olivine (60-90\%) and minor pyroxene which are embedded in a dark matrix of carbonaceous material (graphite) (Mittlefehldt et al., 1998). Olivine is Mg-rich ( $\left.\mathrm{Fo}_{74-95}\right)$ and pyroxene is in equilibrium (Mittlefehldt et al., 1998). Spectra of most ureilites exhibit absorption features near 1 and $2 \mu \mathrm{m}$ due to olivine and pyroxene, but because of the presence of carbon these features are severely suppressed (Band I depth 612\%, Band II depth 1-3\%) (see Fig. 5). Most ureilites have moderate albedos between 0.10-0.14, and spectrally they exhibit flat or negative slopes across the 0.7-2.6 $\mu \mathrm{m}$ interval (Cloutis et al., 2010). In contrast, 246 Asporina has an olivine chemistry of Fo56.5 5 , an albedo of $\sim 0.21$, and a Band I depth of $24 \%$, which are inconsistent with ureilites. Based on the differences in chemistry and band parameters we conclude that ureilites are not analogues for 246 Asporina.

$\mathrm{R}$ chondrites $\left(\mathrm{Fo}_{60-63}\right)$ (Brearley and Jones, 1998) and brachinites $\left(\mathrm{Fo}_{60}-75\right)$ (Mittlefehldt et al., 1998) are olivine-dominated meteorites with chemistries within the range of the Asporina's estimated chemistry. $\mathrm{R}$ chondrites are formed under highly oxidizing nebular processes with most olivine having a chemistry of $\mathrm{Fo}_{60-63}$ with minor clinopyroxene $(\sim 5 \%)$ and traces of lowCa pyroxene (Schulze et al., 1994). Brachinites are formed in igneous processes and contain 8090\% olivine with 5-10\% clinopyroxene and traces of low-Ca pyroxene (Mittlefehldt et al., 2003). Examples of R chondrite and brachinite spectra are depicted in Fig. 5. Based on Asporina's olivine chemistry $\mathrm{F}_{56.5 \pm 5}$, and the lack of a significant $2 \mu \mathrm{m}$ feature due to pyroxene, brachinites are the most probable meteorite analogues for this asteroid, although $\mathrm{R}$ chondrites can not be completely ruled out. The moderate albedo of 0.21 for (246) Asporina also supports this linkage with brachinites, which have relatively moderate albedos of $\sim 0.19$. The albedo of brachinites was estimated from the lab spectra of a $<150 \mu \mathrm{m}$ size powder of Brachina, obtained at the University of Winnipeg HOSERLab for this study, and EET99402 from the RELAB database. Sunshine et al. (2007) modeled the formation of brachinites from an $\mathrm{R}$ chondrite precursor using MELTS model (Asimow and Ghiorso, 1998; Ghiorso and Sack, 1995) and concluded that an olivine-rich residue with Fa concentrations similar to brachinites can be produced at $\sim 25 \%$ melting at a temperature of $\sim 1220^{\circ} \mathrm{C}$. This suggests that Fe-rich olivines like those in brachinites can be created starting with an $\mathrm{R}$ chondrite type material.

For asteroid (289) Nenetta, the temperature corrected Band I center is located at $1.074 \mu \mathrm{m}$ with a Band I depth of $48.08 \%$. Using Eq. (1) we determined that the olivine composition of this asteroid is $\mathrm{Fo}_{48.8 \pm 5}$. This composition, depicted in Fig. 4 as a filled square, is consistent with the range reported by Cruikshank and Hartmann (1984) $\left(\mathrm{Fo}_{40-80}\right)$, and similar to that found by Lucas et al. (2012) (Fo53), however it is lower than the value obtained by Sunshine et al. (2007) $\left(\mathrm{Fo}_{60}\right)$. Based on its olivine chemistry, the lack of a significant $2 \mu \mathrm{m}$ feature, and its albedo of 0.24 , brachinites are the most likely meteorite analogues. However, as in the case of (246) Asporina, R chondrites can not be completely ruled out. It is also important to point out that the olivine composition of (289) Nenetta seems to be more ferroan than the typical compositions observed among brachinites and $\mathrm{R}$ chondrites.

The spectrum of asteroid (354) Eleonora has a Band I center at $1.065 \mu \mathrm{m}$ and Band I depth of $27.04 \%$. For this object we obtained an olivine composition of $\mathrm{Fo}_{66.3 \pm 5}$ (Fig. 目 filled triangle). Sunshine et al. (2007) found that this asteroid is a magnesian object, however their estimated 
composition of Fo92 is much higher than ours. The composition of (354) Eleonora determined by us seems to be consistent with the range found for brachinites $\left(\mathrm{Fo}_{60}-75\right)$.

Asteroid (1951) Lick is the only near-Earth asteroid (NEA) among the studied objects. The Band I center of (1951) Lick is located at $1.063 \mu \mathrm{m}$ (after temperature correction) and its Band I depth has a value of $38.35 \%$. Using MGM, Sunshine et al. (2007) found that the composition of this asteroid is consistent with magnesian olivine ( $\left.\mathrm{Fo}_{79}\right)$. Brunetto et al. (2007) determined that the best fit of Lick's spectrum is obtained by a linear combination of reflectance spectra of San Carlos olivine (20\%) and meteorite Brachina (80\%), along with a fraction of nanophase iron needed to account for space weathering effects. Our analysis of (1951) Lick using Eq.(1) yielded a composition of $\mathrm{Fo}_{70.2 \pm 5}$, which is the highest forsterite value found among the monomineralicolivine asteroids studied in this work. The olivine chemistry of (1951) Lick, represented in Fig. 4 as an open triangle, is consistent with the composition of brachinites.

In the case of asteroid (4125) Lew Allen, the Band I center is located at $1.065 \mu \mathrm{m}$ and the Band I depth is $23.56 \%$. According to our calculations, the olivine chemistry for this asteroid is Fo66.3 \pm 5 (Fig. 4, filled triangle). No previous work was performed on this object. Based on its olivine chemistry and the absence of a significant $2 \mu \mathrm{m}$ feature, brachinites are the best meteorite analogues for this asteroid.

For asteroid (4490) Bambery, the Band I center is located at $1.070 \mu \mathrm{m}$ with a Band I depth of $29.11 \%$. As in the previous cases, the olivine composition of the asteroid was calculated, given the value of $\mathrm{Fo}_{56.5 \pm 5}$ (Fig. 4, filled circle). Like Lew Allen, this is the first time that the composition of this asteroid is determined. Brachinites and $\mathrm{R}$ chondrites have olivine compositions that, within the uncertainty, are consistent with the value found for Bambery. However, considering the lack of the $2 \mu \mathrm{m}$ feature and its albedo of $\sim 0.22$, brachinites are the most probable meteorite analogues for this object.

Asteroid (5261) Eureka is the largest known Mars Trojan (Trilling et al., 2007). Rivkin et al. (2007) obtained NIR spectra of Eureka with the IRTF and SpeX. From their analysis they identified angrites as possible meteorite analogues for this asteroid. Angrites are a rare group of basaltic achondrites with mineralogies dominated by CaO-rich olivine, Ca-Al-Ti-rich pyroxene and anorthite (Weisberg et al., 2006). Spectrally, they exhibit a broad absorption feature centered around $1 \mu \mathrm{m}$ and, in some cases, a weak absorption feature near $2 \mu \mathrm{m}$ (Burbine et al., 2006). Using Hapke formalism, Rivkin et al. (2007) modeled the reflectance spectrum of Eureka by combining angrites and neutral components. Although they obtained a reasonably good fit for Eureka's spectrum they couldn't rule out R chondrites as possible meteorite analogues. The thermal-IR spectrum $(5-30 \mu \mathrm{m})$ of Eureka obtained with the Spitzer IRS by Lim et al. (2011) showed olivine reststrahlen features similar to those observed from laboratory spectra of R chondrites, brachinites and chassignites. From their study Lim et al. (2011) determined that the molar content of Fo for Eureka is probably not higher than Fo65. The measured Band I center for Eureka is located at $1.074 \mu \mathrm{m}$ with a band depth of $27.7 \%$. Using Eq. (1) we calculated its olivine composition, giving a value of $\mathrm{Fo}_{48.8 \pm 5}$ (Fig. 4, filled square). Similar to asteroid (289) Nenetta, the olivine composition of Eureka is more ferroan than the typical compositions found among olivine-rich meteorites. However, based on these results and the lack of a significant $2 \mu \mathrm{m}$ feature, brachinites are the most likely meteorite analogues for Eureka, although $\mathrm{R}$ chondrites can not be completely ruled out.

In general, olivine compositions of asteroids obtained using Eq. (1) seems to be more Fe-rich than those estimated by previous work using MGM (e.g., Sunshine and Pieters, 1998; Sunshine et al., 2007). These differences regarding the estimation of forsterite chemistry based on the Band I center by (e.g., Sunshine and Pieters, 1998; Sunshine et al., 2007) and King and Ridley (1987) are due to the way the band center is calculated. The band centers calculated using MGM are at shorter wavelengths than those of King and Ridley (1987). In addition, the 
temperature correction that we have applied to the asteroid Band I centers using Eq. (3) slightly increases their value, hence the molar content of Fo calculated using Eq. (1) will decrease.

As noted earlier, monomineralic olivine can be formed through different mechanism: accretion of grains from an oxidized nebular region without significant post-accretionary heating $(\mathrm{R}$ chondrites), partial melting with extraction of a basaltic melt leaving an olivine-rich residue (brachinites), or crystallization of olivine from a melt to form an olivine mantle or olivine-rich layer (differentiated object). The Fe content of igneously formed olivine depends on the Fe content of the starting parent material with the Fe content of the final olivine never being higher than the precursor material. For example, olivine produced in an igneous process with an LLchondrite precursor material can never have Fe content more than $\mathrm{Fo}_{72}$. In other words, the only way to produce a Fe-rich olivine via igneous processing is to start with a precursor material that has high Fe content (Sunshine et al., 2007). Based on the results obtained, and taking into account the uncertainties associated to the Band I centers and Fo content, it is possible that at least three of the studied asteroids (354 Eleonora, 1951 Lick and 4125 Lew Allen) could have formed from melting of ordinary chondrite-like bodies.

\subsection{Olivine-rich asteroids}

Asteroids that have been grouped in this category are: (446) Aeternitas, (863) Benkoela, (984) Gretia, (2501) Lohja, and (3819) Robinson. In addition to the $1 \mu \mathrm{m}$ feature, the spectra of these objects exhibit a weak absorption feature at $\sim 2 \mu \mathrm{m}$ indicative of the presence of a second mineral in the assemblages, most likely pyroxene. For each VIS-NIR spectrum spectral band parameters, band centers, Band Area Ratios (BAR), and band depths along with their errors were measured in the same way as in Sanchez et al. (2012, 2013). The band parameters with their corresponding errors are presented in Table 3, The Band I centers and BAR of the olivine-rich asteroids are depicted in Fig. 3 as open circles.

Table 3: Spectral band parameters for the olivine-rich asteroids. The columns in this table correspond to: object number and designation, Band I center $(\mathrm{BIC} \pm 0.005)$, Band I depth $\left(\mathrm{BI}_{\mathrm{depth}} \pm 0.3\right)$, Band II center $(\mathrm{BIIC} \pm 0.01)$, Band II depth $\left(\mathrm{BII}_{\mathrm{depth}} \pm 0.5\right)$, Band Area Ratio (BAR \pm 0.03$)$, olivine-pyroxene abundance ratio $(\mathrm{ol} /(\mathrm{ol}+\mathrm{px}) \pm 0.003)$, the ratio of low-Ca pyroxene to total pyroxene $(\mathrm{LCP} /(\mathrm{LCP}+\mathrm{HCP}) \pm 0.09)$, and geometric albedo $\left(p_{v}\right)$. In the case of the olivine-rich asteroids no temperature corrections to the band parameters were applied because spectra of $\mathrm{R}$ chondrites obtained at different temperatures are not available. Albedo values are obtained from Masiero et al. (2011).

\begin{tabular}{|c|c|c|c|c|c|c|c|c|}
\hline Object & $\mathrm{BIC}(\mu \mathrm{m})$ & $\mathrm{BI}_{\text {depth }}(\%)$ & $\mathrm{BIIC}(\mu \mathrm{m})$ & $\mathrm{BII}_{\mathrm{depth}}(\%)$ & $\mathrm{BAR}$ & $\mathrm{ol} /(\mathrm{ol}+\mathrm{px})$ & $\mathrm{LCP} /(\mathrm{LCP}+\mathrm{HCP})$ & $p_{v}$ \\
\hline 446 Aeternitas & 1.066 & 38.00 & 1.980 & 3.00 & 0.07 & 0.92 & 0.90 & $0.1902 \pm 0.0492$ \\
863 Benkoela & 1.059 & 33.50 & 1.982 & 6.50 & 0.17 & 0.68 & 0.89 & $0.1123 \pm 0.0163$ \\
984 Gretia & 1.045 & 31.64 & 2.040 & 4.77 & 0.14 & 0.78 & 0.60 & $0.3990 \pm 0.0930$ \\
2501 Lohja & 1.061 & 41.90 & 2.022 & 3.20 & 0.06 & 0.93 & 0.69 & $0.1898 \pm 0.0440$ \\
3819 Robinson & 1.055 & 39.20 & 2.026 & 4.35 & 0.08 & 0.91 & 0.67 & $0.3580 \pm 0.2982$ \\
\hline
\end{tabular}

The most common secondary or accessory mineral present in olivine-rich asteroids is low-Ca pyroxene, which gives rise to two absorption features at $\sim 0.9$ and $2 \mu \mathrm{m}$. Depending on the type of pyroxene and its Fe content, the band center of the $1 \mu \mathrm{m}$ olivine feature is shifted to shorter wavelengths. From the calibration developed by Reddy et al. (2011b), the band center of the $1 \mu \mathrm{m}$ olivine feature ranges from $\sim 1.048 \mu \mathrm{m}$ for pure forsterite to $\sim 1.1 \mu \mathrm{m}$ for pure fayalite. Depending on the Fo content of the olivine and the abundance of low-Ca pyroxene in a olivine + low-Ca pyroxene assemblage, the Band I center could be shifted beyond this band center range $(\sim 1.048-1.10 \mu \mathrm{m})$ leading to inconsistent Fo estimates using this calibration. Even if the measured Band I centers are within this range the presence of pyroxene might shift 
Table 4: Spectral band parameters for the $\mathrm{R}$ chondrites. The columns in this table correspond to: meteorite name, RELAB ID, Band I center (BIC \pm 0.003$)$, Band II center (BIIC \pm 0.005$)$, Band Area Ratio $(\mathrm{BAR} \pm 0.01)$, olivine-pyroxene abundance ratio ol $/(\mathrm{ol}+\mathrm{px})$, and the ratio of low-Ca pyroxene to total pyroxene $\mathrm{LCP} /(\mathrm{LCP}+\mathrm{HCP})$, both derived from laboratory measurements.

\begin{tabular}{|c|c|c|c|c|c|c|}
\hline Meteorite & RELAB ID & BIC $(\mu \mathrm{m})$ & BIIC $(\mu \mathrm{m})$ & BAR & ol $/(\mathrm{ol}+\mathrm{px})$ & $\mathrm{LCP} /(\mathrm{LCP}+\mathrm{HCP})$ \\
\hline NWA753 & TB-TJM-114 & 1.061 & 1.951 & 0.08 & - & - \\
LAP04840 & DD-AHT-107 & 1.032 & 1.954 & 0.01 & $0.96^{a}$ & $1.00^{a}$ \\
Rumuruti & MT-TJM-013 & 1.067 & 2.121 & 0.06 & $0.93^{b}$ & $0.09^{b}$ \\
PRE95411 & MT-TXH-045 & 1.066 & 1.978 & 0.20 & - & - \\
PCA91002 & MB-TXH-065-A & 1.065 & 2.072 & 0.13 & $0.81^{c}$ & $0.57^{c}$ \\
ALH85151 & MB-TXH-045 & 1.068 & 2.060 & 0.17 & $0.68^{d}$ & $0.53^{d}$ \\
A-881988 & MP-TXH-059 & 1.051 & 1.968 & 0.03 & - & - \\
\hline
\end{tabular}

a Data from McCanta et al. (2008)

${ }^{b}$ Data from Schulze et al. (1994)

${ }^{c}$ Data from Rubin and Kallemevn 1994)

${ }^{d}$ Data from Kallemevn et al. (1996)

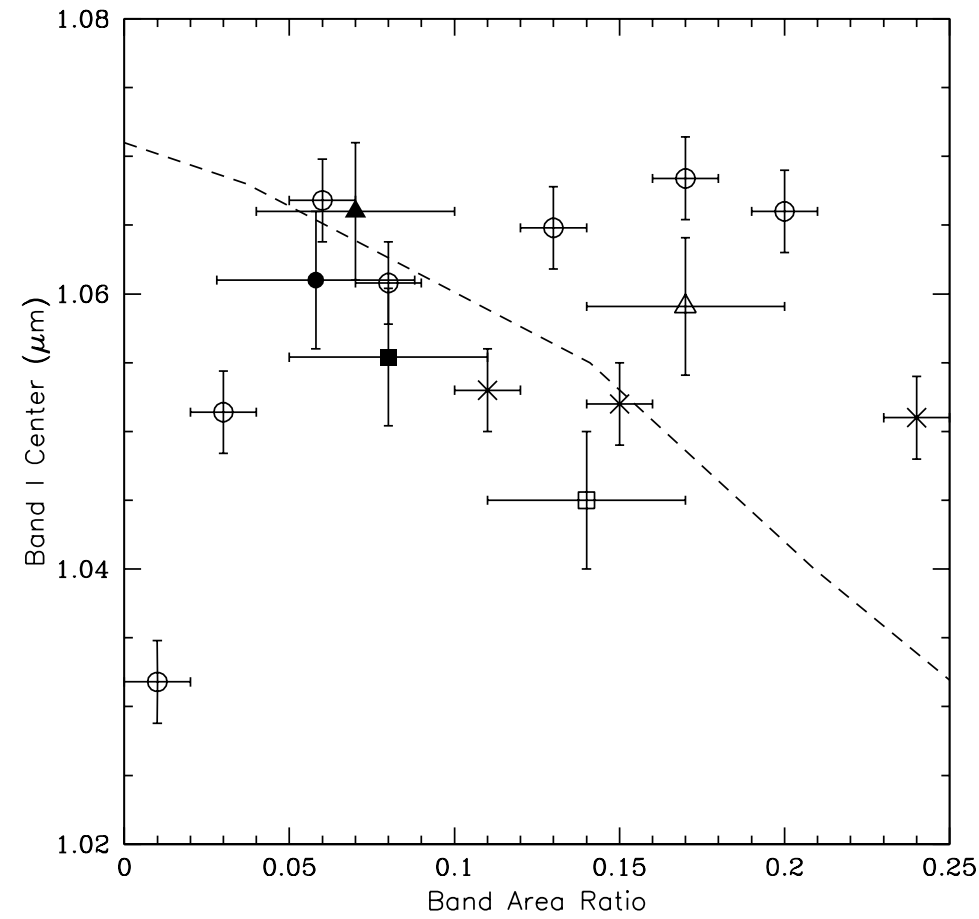

Figure 6: Band I center vs. Band Area Ratio (BAR) for olivine-rich asteroids and R chondrites. Values measured for $\mathrm{R}$ chondrites are depicted with open circles. The asteroids whose band parameters have been plotted are: (2501) Lohja (filled circle), (3819) Robinson (filled square), (446) Aeternitas (filled triangle), 863 Benkoela (open triangle) and (984) Gretia (open square). For comparison we have included measured values (x symbols) from a mixture of OLV003 (forsterite)+PYX016 (diopside) obtained at the University of Winnipeg HOSERLab. The dashed curve indicates the location of the olivine-orthopyroxene mixing line from Cloutis et al. (1986).

the Band I center to longer or shorter wavelength (depending on the type of pyroxene and the cation abundance) preventing an accurate determination of olivine chemistry using Eq. (1). 


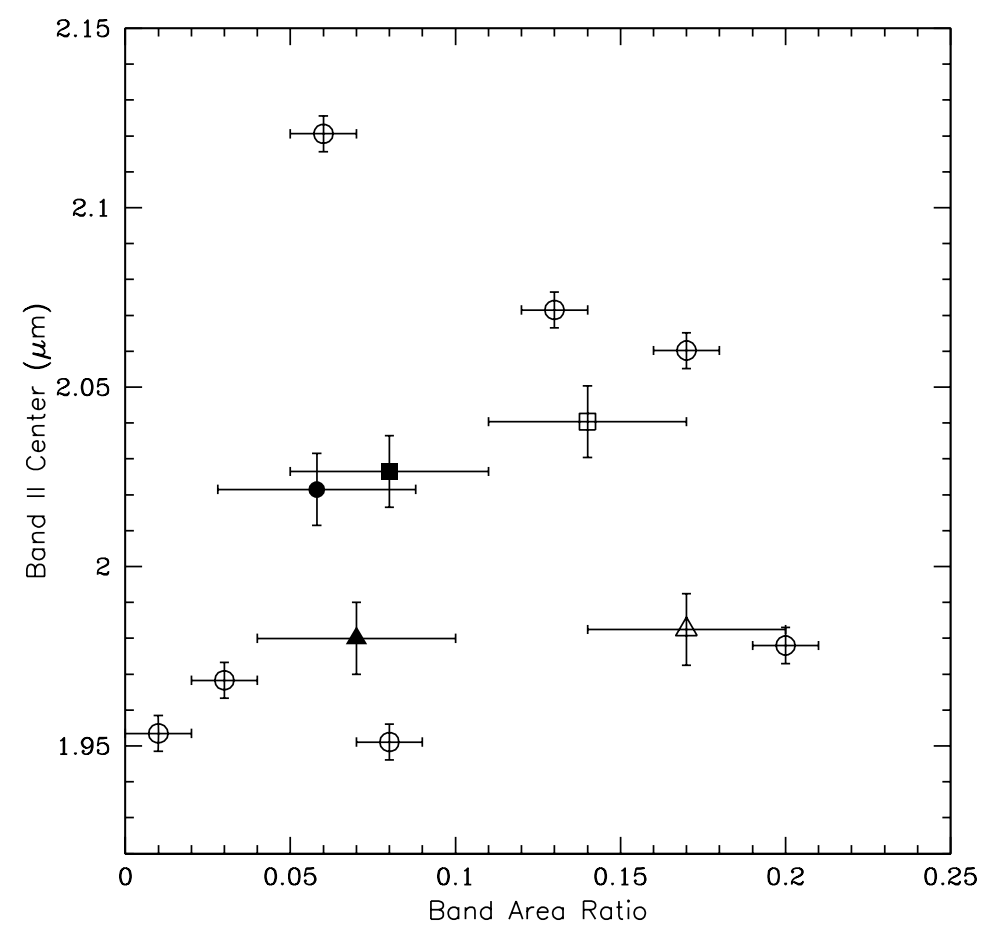

Figure 7: Band II center vs. Band Area Ratio (BAR) for olivine-rich asteroids and R chondrites. Values measured for $\mathrm{R}$ chondrites are depicted with open circles. The asteroids whose band parameters have been plotted are: (2501) Lohja (filled circle), (3819) Robinson (filled square), (446) Aeternitas (filled triangle), 863 Benkoela (open triangle) and (984) Gretia (open square).

Therefore, we decided not to use this calibration with the olivine-rich asteroids and to look for possible meteorite analogs that could be used to develop a new calibration.

Olivine-rich meteorites that could be potential analogs for these asteroids include pyroxene pallasites, brachinites, ureilites, and R-chondrites. Pyroxene pallasites are dominated by olivine (55-63 vol \%) and contain (1-3 vol \%) low-Ca pyroxenes (Mittlefehldt et al., 1998). Due to the presence of metal, sample preparation for laboratory spectroscopy is extremely difficult for metal-silicate mixtures like pallasites (Gaffey, 1976; Cloutis et al., 1990). As a result there are no useful NIR laboratory spectra of both metal and olivine+pyroxene from a pyroxene pallasite. Due to this limitation, these meteorites are not considered in the present analysis. As it was mentioned earlier, brachinites contain $\sim 5-10 \%$ clinopyroxene and traces of low-Ca pyroxene, however brachinite spectra don't show the $2 \mu \mathrm{m}$ feature. Spectra of most ureilites show absorption features near 1 and $2 \mu \mathrm{m}$, however these features are severely suppressed. Band depths measured for the olivine-rich asteroids in our sample range from $\sim 32-42 \%$ (Band I depth), and from $\sim 3-7 \%$ (Band II depth). Furthermore, the typical albedos for ureilites $(0.10-$ 0.14) are lower than most of olivine-rich asteroids. R chondrites, on the other hand, have an olivine abundance of typically $65-78 \mathrm{vol} \%$ and different proportions of low-Ca and Ca-pyroxene (Bischoff et al., 2011). Spectrally they show the $1 \mu \mathrm{m}$ feature and a weak absorption feature at $\sim 2 \mu \mathrm{m}$. In Figs. 6 and 7 we plotted the Band I and Band II centers vs. BAR, respectively of seven $\mathrm{R}$ chondrite spectra from the RELAB database. Measured values are depicted as open circles and have been plotted along with the values measured from the olivine-rich asteroid spectra. Spectral band parameters of the $\mathrm{R}$ chondrites are presented in Table 4 . An inspection of Figs. 6 and 7 shows that the band centers and BAR values of the olivine-rich asteroids are 
located within the range of those measured for the $\mathrm{R}$ chondrites. As can be seen in Fig. 6, values obtained from the $\mathrm{R}$ chondrites deviate from the olivine-orthopyroxene mixing line of Cloutis et al. (1986) (dashed curve). This deviation could be explained by the presence of a clinopyroxene component in the $\mathrm{R}$ chondrites. This behavior is also observed in the measured values of an olivine-clinopyroxene mixture depicted as " $x$ " symbols in Fig. 6. These values correspond to a mixture in which the fraction of olivine to clinopyroxene ranges from 80:20 to 40:60. Based on these results we developed a new set of calibrations in an effort to determine the olivine abundance and the ratio of low-Ca pyroxene to total pyroxene of the olivine-rich asteroids.

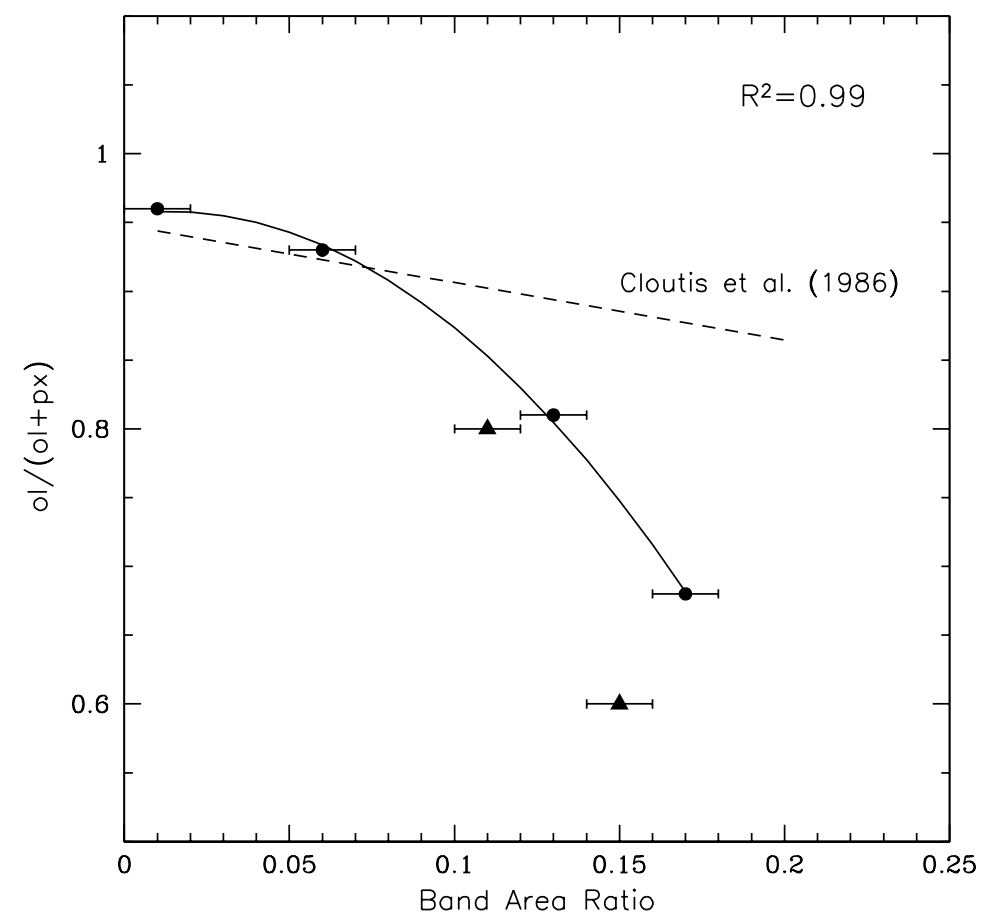

Figure 8: ol/(ol+px) ratios vs. Band Area Ratio (BAR) measured for $\mathrm{R}$ chondrites (filled circles). The solid line represents a second order polynomial fitted to the data. The coefficient of determination $\left(\mathrm{R}^{2}\right)$ is given. The root mean square error between the $\mathrm{ol} /(\mathrm{ol}+\mathrm{px})$ ratios determined using Eq. (4) and the laboratory measurements is 0.003. For comparison we have included the linear relationship between $\mathrm{ol} /(\mathrm{ol}+\mathrm{opx})$ and BAR from Cloutis et al. (1986) (dashed line) given by $o l /(o l+o p x)=-0.417 \times B A R+$ 0.948. Also shown, as filled triangles, measured values from a mixture of OLV003 (forsterite) +PYX016 (diopside) obtained at the University of Winnipeg HOSERLab.

Figure 8 shows the $\mathrm{ol} /(\mathrm{ol}+\mathrm{px})$ ratios vs. BAR measured for $\mathrm{R}$ chondrites. Here the $\mathrm{ol} /(\mathrm{ol}+\mathrm{px})$ values correspond to the abundances of olivine and LCP and HCP. Modal abundances (included in Table 4) are only available for four samples, however from Fig. 8 it is possible to see how the $\mathrm{ol} /(\mathrm{ol}+\mathrm{px})$ ratio decreases as the BAR increases. This curve (solid line) shows a much steeper decrease in olivine content with increasing BAR than shown by the Cloutis et al. (1986) olivine pyroxene mixing experiments (dashed line). This discrepancy could be attributed to the fact that the linear relationship obtained by Cloutis et al. (1986) was derived from an olivine-orthopyroxene mixture, while our measurements include both LCP and HCP. As an example we have included in Fig. 8 measured values from an olivine-clinopyroxene mixture (depicted as filled triangles), which show a pronounced deviation from the linear rela- 
tionship obtained by Cloutis et al. (1986). The correlation between the ol/(ol+px) ratio and the BAR found for the $\mathrm{R}$ chondrites can be described by a second order polynomial fit:

$$
o l /(o l+p x)=-11.27 \times B A R^{2}+0.302 \times B A R+0.956
$$

With this equation we determined the olivine abundance of the olivine-rich asteroids from their BAR values. Calculated ol $/(\mathrm{ol}+\mathrm{px})$ ratios are presented in Table 3 ,

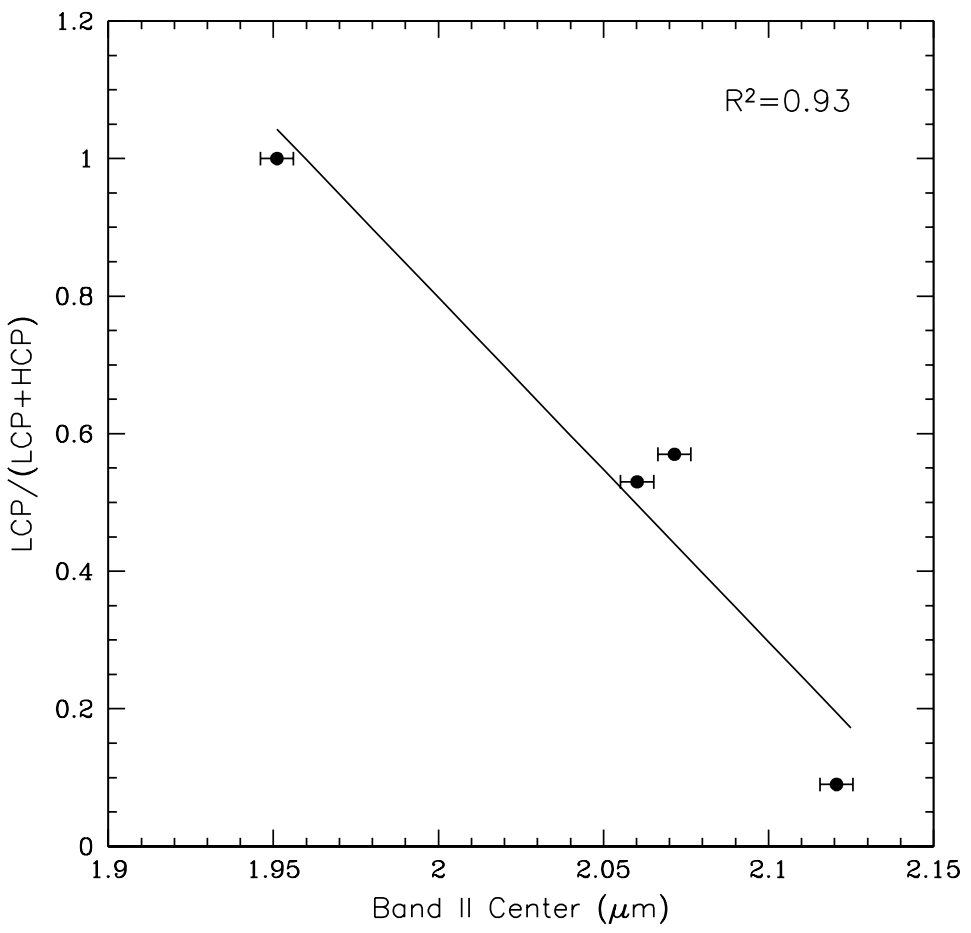

Figure 9: $\mathrm{LCP} /(\mathrm{LCP}+\mathrm{HCP})$ ratios vs. Band II center measured for $\mathrm{R}$ chondrites. The solid line represents a linear fit to the data. The coefficient of determination $\left(\mathrm{R}^{2}\right)$ is given. The root mean square error between the $(\mathrm{LCP} /(\mathrm{LCP}+\mathrm{HCP}))$ ratios determined using Eq. (5) and the laboratory measurements is 0.09.

In Figure 9 we plotted $\mathrm{LCP} /(\mathrm{LCP}+\mathrm{HCP})$ ratios vs. Band II centers measured for $\mathrm{R}$ chondrites. Here the $\mathrm{LCP} /(\mathrm{LCP}+\mathrm{HCP})$ values correspond to the ratio of low-Ca pyroxene to total pyroxene. As can be seen in Fig. 9, Band II centers move to longer wavelengths with increasing $\mathrm{Ca}^{2+}$ content. This shift in the wavelength position is explained by crystal field theory (CFT), and is due to the effects of replacing the larger $\mathrm{Ca}^{2+}$ cation into the crystallographic site of the smaller $\mathrm{Fe}^{2+}$ cation, resulting in changes in the crystal structure and thus in the crystal fields and electronic transitions which produce this absorption feature (Burns, 1993). A least-squares fit of the data in Fig. 9 yields

$$
L C P /(L C P+H C P)=-5.006 \times(B I I C)+10.81
$$

Using equation (5) we determined the fraction of low-Ca pyroxene present in the olivine-rich asteroids from their measured Band II centers. The LCP/ $(\mathrm{LCP}+\mathrm{HCP})$ ratios are presented in Table 3. In the case of the olivine-rich asteroids no temperature corrections were derived since spectra of $\mathrm{R}$ chondrites obtained at different temperatures are not available. It is important to stress that the results obtained using Eqs. (4) and (5) must be taken with caution. Although 
$\mathrm{R}$ chondrites provide a good spectral match for olivine-rich asteroids, their compositions might differ. In addition, these equations were derived from a very limited sample. Thus, these calculations represent only a first attempt to determine mineral abundances for this type of asteroids. Additional laboratory measurements of meteorite samples will be required to improve these spectral calibrations.

\subsubsection{Mineralogical analysis}

Of all the S(I)-type asteroids, (446) Aeternitas is one of the best-characterized members. Based on spectral analysis, Cloutis et al. (1990) suggested that the surface assemblage of (446) Aeternitas contained roughly $35 \mathrm{wt} \%$ metal, $55 \mathrm{wt} \%$ olivine $\left(\mathrm{Fo}_{80 \pm 10}\right), 7 \mathrm{wt} \%$ clinopyroxene, and $3 \mathrm{wt} \%$ orthopyroxene. Similar to (246) Asporina (Cruikshank and Hartmann, 1984), Cloutis et al. (1990) suggested a substantial fine-grained $(<45 \mu \mathrm{m})$ component on the surface and concluded that the best match for the spectrum of this asteroid is fine-grained olivine scattered on a roughened metal surface. Hiroi and Sasaki (2001) modeled the reflectance spectrum of Aeternitas using model mixtures of minerals that were irradiated with a pulse-laser that simulates the space weathering effect. They found that the best fit for Aeternita's spectrum was obtained combining $2 \%$ fresh olivine, $93 \%$ space-weathered olivine, $1 \%$ space-weathered orthopyroxene, and 4\% chromite. Sunshine et al. (2007) suggested that (446) Aeternitas is magnesian with $\sim 5-10 \%$ pyroxene based on the presence of a weak $2 \mu \mathrm{m}$ feature. The VIS-NIR spectrum of Aeternitas exhibits a broad, asymmetric feature (Band I depth 38\%) indicating the presence of olivine as the primary mineral on the surface. The calculated Band I center is 1.066 $\mu \mathrm{m}$. There is also a weak $\sim 3 \%$ feature centered at $1.98 \mu \mathrm{m}$ and the BAR is 0.07 . From its BAR value and using Eq. (4) we determined that the $\mathrm{ol} /(\mathrm{ol}+\mathrm{px})$ ratio for Aeternitas is 0.92 . From the measured Band II center we estimated a $\mathrm{LCP} /(\mathrm{LCP}+\mathrm{HCP})$ ratio value of 0.90 .

The spectrum of asteroid (863) Benkoela exhibits two absorption features centered at 1.059 $\mu \mathrm{m}$ (Band I depth 33.5\%) and $1.982 \mu \mathrm{m}$ (Band II depth 6.5\%), with a BAR value of 0.17. For this asteroid we determined an olivine abundance of 0.68 , the lowest among the olivine-rich asteroids studied, and a ratio of low-Ca pyroxene to total pyroxene of 0.89 .

For (984) Gretia, the Band I center is located at $1.045 \mu \mathrm{m}$ and the Band II center is at $2.040 \mu \mathrm{m}$ with a BAR of 0.14 . Band I and II depths are $31.64 \%$ and $4.77 \%$, respectively. Using Eqs. (4) and (5) we found that the ol $/(\mathrm{ol}+\mathrm{px})$ ratio for this asteroid is 0.78 and the $\mathrm{LCP} /(\mathrm{LCP}+\mathrm{HCP})$ ratio is 0.60 , implying that Gretia has the lowest fraction low-Ca pyroxene among the studied asteroids.

The measured Band I and Band II centers of (2501) Lohja are located at $1.061 \mu \mathrm{m}$ (Band I depth $41.9 \%$ ) and $2.022 \mu \mathrm{m}$ (Band II depth 3.2\%), respectively. The calculated BAR is 0.06 . With an ol/ $(\mathrm{ol}+\mathrm{px})$ ratio of 0.93 , Lohja has the highest olivine abundance among the olivine-rich asteroids studied. The fraction of low-Ca pyroxene was estimated to be 0.69.

Like the other olivine-rich asteroids, the spectrum of (3819) Robinson exhibits two absorption features, one centered at $1.055 \mu \mathrm{m}$ with a Band I depth of $39.20 \%$, and the other centered at $2.026 \mu \mathrm{m}$ with a Band II depth of $4.35 \%$. The calculated BAR is 0.08 . From its BAR value and using Eq. (4) we found that the ol/ $(\mathrm{ol}+\mathrm{px})$ ratio for Robinson is 0.91 . The ratio of low-Ca pyroxene to total pyroxene gave a value of 0.67 .

Based on spectral matching Sunshine et al. (2007) suggested that the olivine composition of these olivine-rich asteroids is more likely to be MgO-rich. According to them this magnesian composition along with the position of the pyroxene feature $(<2.2 \mu \mathrm{m})$, indicative of the presence of low-Ca pyroxene, would suggest that these objects formed from melting of ordinary chondrite material. This would make pallasites their most likely meteorite analogue. Our results suggest that $\mathrm{R}$ chondrites are better meteorite analogues for these asteroids. However, further analysis of meteorite samples will be needed to support or reject this idea. 


\section{Dynamical connection with asteroid families}

In addition to the mineralogical analysis, an important part in understanding the formation of S(I)-type asteroids is to try to establish dynamical links between these objects and other asteroids or families. Some asteroid families show compositional diversity among their members. This diversity could be explained by the presence of interlopers in the family, but could also reflect the compositional gradient of what once was a differentiated asteroid later fragmented. Although conclusive evidence for the latter scenario still remains elusive, this hypothesis could not be ruled out in recent studies of asteroid families (e.g., Mothé-Diniz et al., 2008). Thus, finding dynamical links between S(I)-type asteroids and metallic and/or basaltic asteroids (representing the core and crust of a differentiated body) could support the scenario in which these olivine-dominated asteroids originated from a differentiated object. Table 5includes the orbital elements and absolute magnitudes of the S(I)-type asteroids studied. After doing a careful inspection of the proper orbital elements $\left(e_{P}, i_{P}, a_{P}\right)$ of asteroids $246,289,354,446,863,984$, 2501 , and 3819 we determined that none of these bodies are related to known families. In particular we compared the orbital elements of these asteroids with those from Nesvorny (2012). The proper eccentricity $\left(e_{P}\right)$ and proper inclination $\left(i_{P}\right)$ versus proper semimajor axis $\left(a_{P}\right)$ of these objects, along with the asteroid families identified by Nesvornv (2012), and background objects are shown in Fig. 10 as filled triangles, red, and blue points, respectively. This result is in agreement with the work of Masiero et al. (2013), in which they linked $\sim 38300$ asteroids into 76 distinct families using the Hierarchical Clustering Method (HCM) and WISE/NEOWISE data. None of the $\mathrm{S}(\mathrm{I})$-type asteroids listed above were linked to the dynamical families of Masiero et al. (2013).

Table 5: Osculating elements and absolute magnitudes of the S(I)-type asteroids studied.

\begin{tabular}{|c|c|c|c|c|}
\hline Object & Semimajor axis (AU) & Eccentricity & Inclination $\left(^{\circ}\right)$ & Absolute magnitude \\
\hline 246 Asporina & 2.6975 & 0.1073 & 15.6265 & 8.62 \\
289 Nenetta & 2.8719 & 0.2058 & 6.6972 & 9.51 \\
354 Eleonora & 2.7985 & 0.1147 & 18.3925 & 6.44 \\
446 Aeternitas & 2.7901 & 0.1261 & 10.6294 & 8.90 \\
863 Benkoela & 3.1986 & 0.0347 & 25.3963 & 9.02 \\
984 Gretia & 2.8032 & 0.1964 & 9.0860 & 9.03 \\
1951 Lick & 1.3905 & 0.0616 & 39.0897 & 14.7 \\
2501 Lohja & 2.4244 & 0.1945 & 3.3129 & 12.08 \\
3819 Robinson & 2.7734 & 0.1363 & 11.0977 & 11.8 \\
4125 Lew Allen & 1.9214 & 0.1178 & 20.4399 & 13.5 \\
4490 Bambery & 1.9311 & 0.0921 & 26.1157 & 12.7 \\
5261 Eureka & 1.5236 & 0.0648 & 20.2810 & 16.1 \\
\hline
\end{tabular}

Two of the studied asteroids, (4125) Lew Allen and (4490) Bambery, have orbital elements which place them in the same region of the Hungaria asteroid family, defined by a semimajor axis of $1.78<a<2.0 \mathrm{AU}$, an eccentricity $e<0.18$, and inclination $16^{\circ}<i<34^{\circ}$ (Warner et al., 2009). Figure 11 shows the Hungaria family in a plot of absolute magnitude $(\mathrm{H})$ versus proper semimajor axis $\left(a_{P}\right)$ from Warner et al. (2009). The location of (434) Hungaria, (4125) Lew Allen, and (4490) Bambery are depicted as a filled triangle, filled square and filled circle, respectively. Asteroid (434) Hungaria has been classified as E/Xe-type (Tholen, 1984; DeMeo et al., 2009) and it has a high geometric albedo of $p_{v}=0.428$. The VIS-NIR spectrum of this asteroid exhibits a few weak absorption features in the visible region $(\sim 0.45-0.9 \mu \mathrm{m})$, and it is relatively smooth and featureless in the wavelength range of $0.8-2.5 \mu \mathrm{m}$ Kelley and Gaffey, 

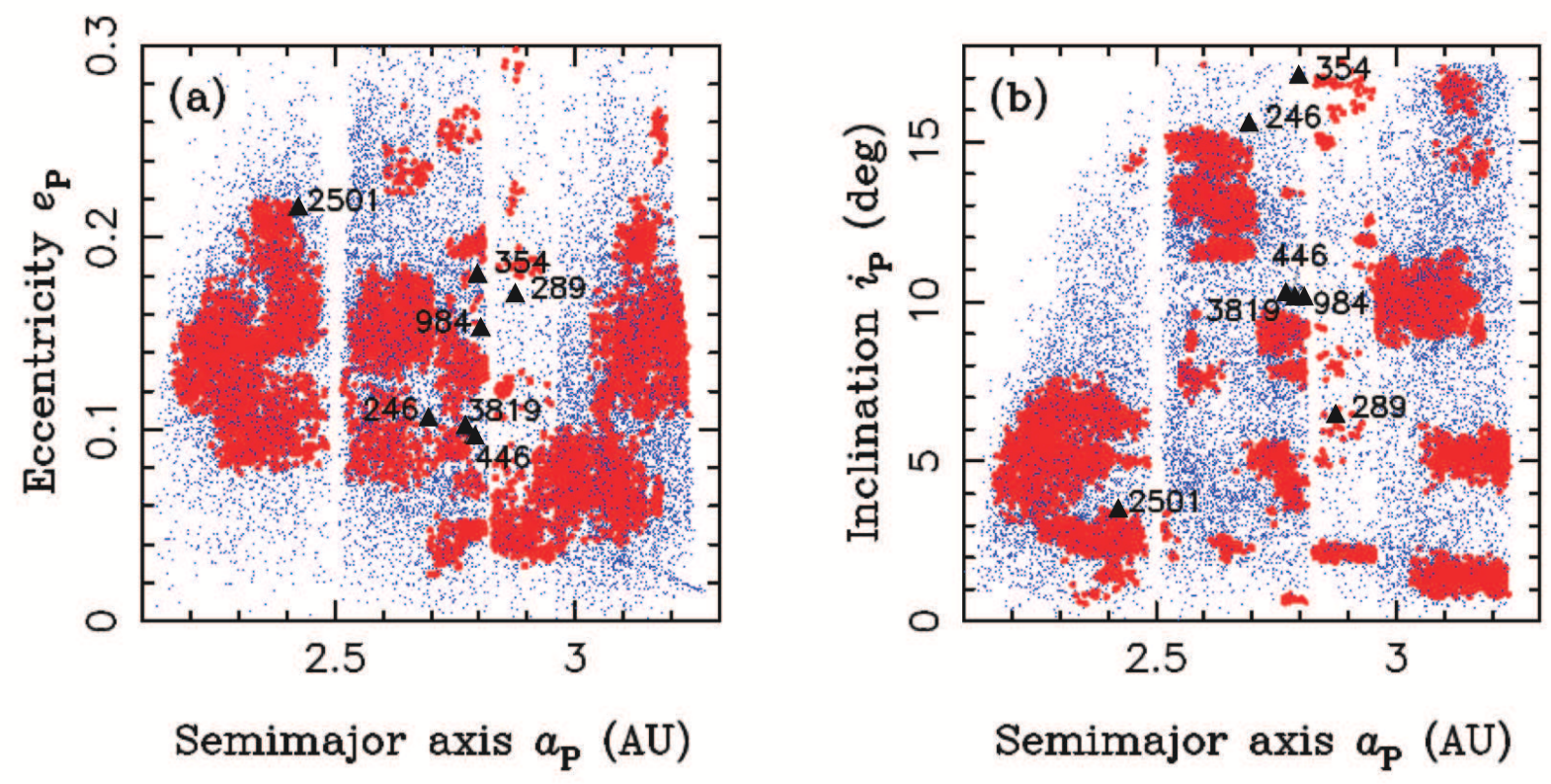

Figure 10: Proper eccentricity $\left(e_{P}\right)$ and proper inclination $\left(i_{P}\right)$ vs. proper semimajor axis $\left(a_{P}\right)$ for the asteroid families identified by Nesvorny (2012) (red), background objects (blue), and the S(I)-type asteroids: 246, 289, 354, 446, 984, 2501, and 3819 (filled triangles). Due to its high inclination, asteroid (863) Benkoela is not included in this figure.

2002). A detailed analysis of (434) Hungaria carried out by Kelley and Gaffey (2002) showed that the surface of this asteroid is mainly composed of iron-free pyroxene, enstatite, making enstatite achondrites (aubrites) the best meteorite analogs for this object. Similar results were obtained by Clark et al. (2004). The only way to produce an object of the size $(\sim 11 \mathrm{~km})$ and composition of Hungaria is through the melting and differentiation of a parent body with an enstatite chondrite composition (Keil, 1989; McCoy et al., 1999; Kelley and Gaffey, 2002). However, this process would lead to the formation of an enstatite mantle instead of an olivine mantle. Therefore, if (434) Hungaria is a fragment of the enstatite mantle of a disrupted body (as suggested by the evidence), a genetic connection between this asteroid and (4125) Lew Allen and (4490) Bambery could be ruled out. Implying that these asteroids are probably interlopers in the Hungaria family.

Asteroid (5261) Eureka is one of the eight Mars Trojans known to date (de la Fuente Marcos and de la Fuente Marcos, 2013). Seven of these objects (including Eureka) are located in the $\mathrm{L}_{5}$ point and one of them in the $\mathrm{L}_{4}$. Possible formation scenarios for Eureka and the Mars Trojans include: collisional fragmentation, rotational fission of the parent body, and capture events in the Trojan region (Christou, 2013; de la Fuente Marcos and de la Fuente Marcos, 2013). Only for three of these asteroids, 5261 Eureka (L $\mathrm{L}_{5}$ point), 101429 (1998 VF31) (L $\mathrm{L}_{5}$ point), and 121514 (1999 UJ7) (L $\mathrm{L}_{4}$ point), spectroscopic observations are available. VIS-NIR spectra $(\sim 0.4-2.5 \mu \mathrm{m})$ of asteroid 101429 (1998 VF31) obtained by Rivkin et al. (2007) showed that this object belongs to the S(VII) subclass defined by Gaffey et al. (1993). Objects in this class are considered to be analogous to the mesosiderite meteorites (i.e., metal-basaltic assemblages). From their analysis, Rivkin et al. (2007) determined that the spectrum of 101429 (1998 VF31) is consistent with a mixture of primitive achondrites and iron, and in general, their mixture models produced good 


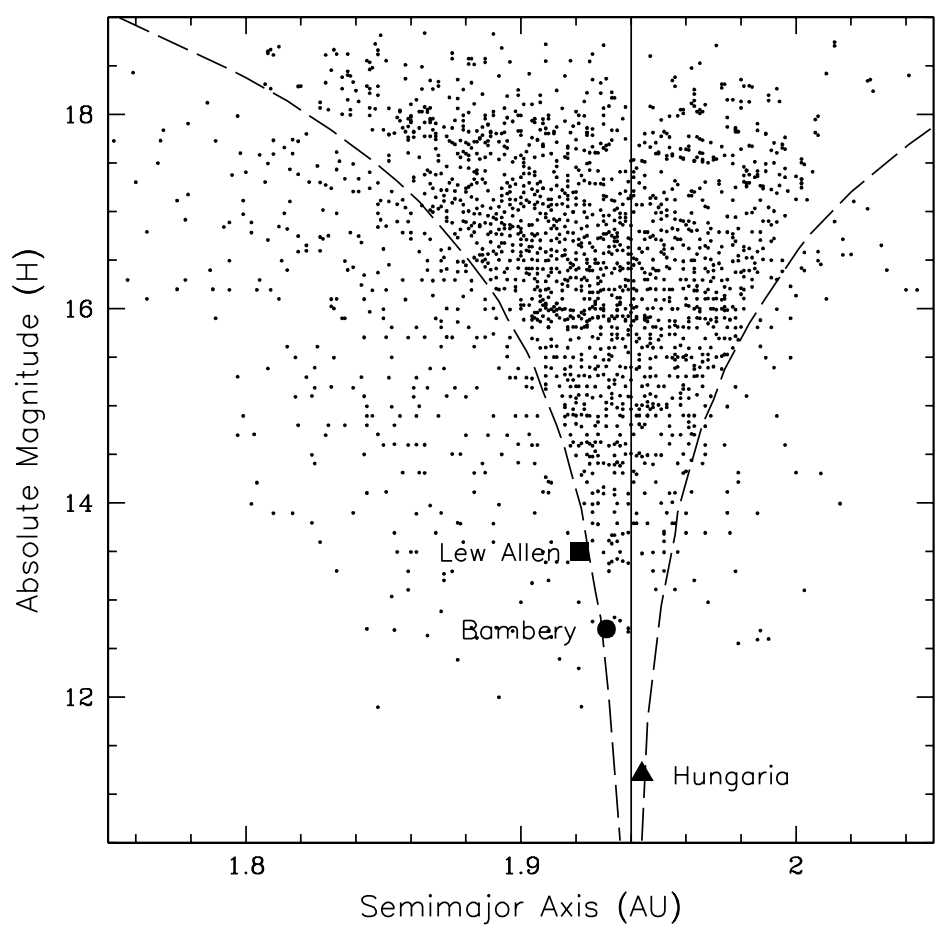

Figure 11: Hungaria-population asteroids in a diagram of absolute magnitude $(\mathrm{H})$ versus proper semimajor axis $\left(a_{P}\right)$ from Warner et al. (2009). The dashed curved lines show the Hungaria-family region where the highest concentration of the asteroids is located. The vertical line represents the center of the collisionally-derived family (Warner et al., 2009). The location of (434) Hungaria, (4125) Lew Allen, and (4490) Bambery are depicted as a filled triangle, filled square and filled circle, respectively.

fits with and without the contribution of mesosiderites. Based on these results, Rivkin et al. (2007) concluded that there is no genetic connection between Eureka and 101429 (1998 VF31), and suggested that at least one of these objects was probably captured into its current orbit during the early stages of the solar system. These results are confirmed by recent numerical models carried out by de la Fuente Marcos and de la Fuente Marcos (2013). VIS spectra (0.5$1.0 \mu \mathrm{m}$ ) of asteroid 121514 (1999 UJ7) acquired by Rivkin et al. (2003) allowed them to classify this object as an X-class (Bus, 1999). Spectra of this class are characterized by being featureless with moderately reddish slopes, and have been associated with iron meteorites and enstatite achondrites (Bus and Binzel, 2002a). Unfortunately, the lack of NIR spectra for 121514 (1999 UJ7) prevents a more detailed mineralogical analysis. Based on the current data it is not possible to establish whether (5261) Eureka is indeed a fragment of the mantle of a primordial body, presumably formed in the terrestrial planets region, or whether it reached its current location during a capture event. Additional spectroscopic observation of the rest of the Mars Trojans could help to constrain the formation scenario for this object.

Asteroid (1951) Lick has a perihelion distance of $q=1.305 \mathrm{AU}$, which places it in the borderline between NEAs $(q \leq 1.3 \mathrm{AU})$ and Mars-crossing asteroids $(1.3<q<1.66 \mathrm{AU})$. Dynamical modeling performed by Dunn et al. (2013) showed that the source regions for olivine-dominated NEAs include: the intermediate source Mars-crossing region, the $\nu_{6}$ secular resonance, and the 3:1 mean-motion resonance with Jupiter. Apart from (1951) Lick, the only other olivinedominated NEA with known composition is (136617) 1994CC (Reddy et al., 2011b). The analysis of this asteroid carried out by (Reddy et al., 2011b) revealed a Mg-rich (Fogo) olivine com- 
position, similar to Mg-rich pallasites with a low metal component. This difference in olivine chemistry between (136617) 1994CC and (1951) Lick (Fo70 5 ), along with the significant difference in their orbital elements suggest that there is no genetic connection between these two objects.

\section{Conclusions}

The surfaces of S(I)-type asteroids are dominated by olivine and in some cases pyroxene as a minor phase. Using VIS-NIR spectroscopy, mineralogy, and mineral chemistry of monomineralic-olivine asteroids can be constrained using olivine spectral calibration developed by King and Ridley (1987) and improved by Reddy et al. (2011b). We have found that the olivine composition for the monomineralic-olivine asteroids ranges from $\sim \mathrm{Fo}_{49}$ to $\mathrm{Fo}_{70}$. Based on their olivine chemistry, albedo, and the absence of a significant $2 \mu \mathrm{m}$ feature we determined that the most plausible meteorite analogues for (354) Eleonora, (1951) Lick and (4125) Lew Allen are brachinites. In the case of (246) Asporina, (289) Nenetta, (4490) Bambery and (5261) Eureka, brachinites are also the most likely meteorite analogues, although $\mathrm{R}$ chondrites can not be completely ruled out.

For the olivine-rich asteroids we found similarities between their spectral band parameters and those measured for $\mathrm{R}$ chondrites. Therefore, we have developed a new set of spectral calibrations from the analysis of VIS-NIR spectra of $\mathrm{R}$ chondrites, whose modal abundances are known. We have established a relationship between the $\mathrm{ol} /(\mathrm{ol}+\mathrm{px})$ ratio and $\mathrm{BAR}$, and the $\mathrm{LCP} /(\mathrm{LCP}+\mathrm{HCP})$ ratio and the Band II centers. Using these equations we have constrained the olivine and low-Ca pyroxene abundance of these objects. In particular, we found that the olivine abundance for the olivine-rich asteroids varies from 0.68 (863 Benkoela) to 0.93 (2501 Lohja). The highest fraction of low-Ca pyroxene (0.9) was found for (446) Aeternitas, while the lowest (0.6) corresponds to (984) Gretia. Although this is a preliminary estimation, this is the first time that the mineral abundance of these asteroids has been determined. More laboratory measurements including both, spectra and modal abundances will be needed to improve the spectral calibrations. These additional data could also help to develop new equations to estimate the olivine and pyroxene chemistry of this type of asteroid. This would be particularly useful considering that the current calibrations (Sunshine et al., 1990; King and Ridley, 1987) are affected by the presence of pyroxene, and therefore can not be used when this mineral is present as a secondary phase.

Based on the spectral and mineralogical characteristics of the studied asteroids, the two most plausible meteorite analogues are brachinites and $\mathrm{R}$ chondrites, which span the primitivedifferentiated range. This suggests that olivine could be produced via nebular processes or in a thermally evolved environment. However, it is also possible that samples of S(I)-type asteroids have not yet been found in terrestrial meteorite collections.

A possible explanation for the paucity of olivine-dominated asteroids in the main belt is that all differentiated asteroids (with the exception of Vesta) were either disrupted or fragmented into pieces during the early stages of the solar system. These fragments, some from the crust and some from the mantle, were subsequently eroded by continuous collisions, being reduced to pieces that are below our current detection limits (see Burbine et al., 1996, and references therein). Our failure in finding dynamical links between the S(I)-type asteroids and basaltic objects could support this hypothesis. On the other hand, depending on the starting material, the melting and differentiation of the parent body could form a mantle with a different composition. As discussed in the previous section, there is evidence that shows that differentiation of an enstatite chondrite-like body will produce an enstatite mantle instead of an olivine mantle. 
Another possible scenario is that the largest S(I)-type asteroids were originally part of differentiated objects that started their lives outside the main belt. In this circumstance, their parent body or bodies were disrupted by early solar system collisions, with the fragments scattered across the inner solar system. We speculate that this led a few S(I)-types to be captured within the primordial main belt by early dynamical processes.

In the existing literature, there are several possible ways $\mathrm{S}(\mathrm{I})$-type asteroids (and other objects) may be captured within the primordial main belt. First, it is possible these bodies reached their current orbits by being scattering into the primordial main belt via interacting planetary embryos (Bottke et al., 2006). Second, the S(I)-types may have been injected into the main belt by the dynamical conditions surrounding the so-called "Grand Tack" (Walsh et al., 2011). In this scenario, Jupiter migrates both inward and outward across the primordial main belt via interactions with the solar nebula. This may have allowed objects in the inner solar system to become deeply embedded within the primordial main belt. A third possibility is that the S(I)-types were captured within the fossil resonances of Jupiter before the so-called Nice model events took place (Bottke and Asphaug, 2013). Fourth, it is plausible they were somehow captured during the events surrounding the Nice model itself (Tsiganis et al., 2005). This last mechanism seems less likely to us as of this writing, however, because the resonances that sweep across the main belt during the Nice model seem more likely to deliver comet-like bodies to the outer main belt than fragments from differentiated bodies (Levison et al., 2009).

Regardless of the exact mechanism, if one or more of these capture scenarios are true in the broadest sense, it seems likely that the S(I)-types were once part of differentiated planetesimals that started their lives in the terrestrial planet region (Bottke et al., 2006). This makes their origin and evolution history extraordinarily interesting from the perspective of understanding terrestrial planet formation.

\section{Acknowledgements}

This paper is based on data obtained with the Infrared Telescope Facility on Mauna Kea, Hawai'i. Some of the data used in this work were obtained from the SMASS II and $\mathrm{S}^{3} \mathrm{OS}^{2}$. In addition, part of the data utilized in this publication were obtained and made available by the MIT-UH-IRTF Joint Campaign for NEO Reconnaissance. The IRTF is operated by the University of Hawaii under Cooperative Agreement no. NCC 5-538 with the National Aeronautics and Space Administration, Office of Space Science, Planetary Astronomy Program. The MIT component of this work is supported by NASA grant 09-NEOO009-0001, and by the National Science Foundation under Grants Nos. 0506716 and 0907766. Any opinions, findings, and conclusions or recommendations expressed in this material are those of the author(s) and do not necessarily reflect the views of NASA or the National Science Foundation. This publication makes use of data products from the Wide-field Infrared Survey Explorer, which is a joint project of the University of California, Los Angeles, and the Jet Propulsion Laboratory/California Institute of Technology, funded by the National Aeronautics and Space Administration. This publication also makes use of data products from NEOWISE, which is a project of the Jet Propulsion Laboratory/California Institute of Technology, funded by the Planetary Science Division of the National Aeronautics and Space Administration. The authors thank, John Hinrichs and Paul Lucey for providing us with data for this research. VR and MJG research was supported by NASA 182 NEOO Program Grant NNX12AG12G, and NASA Planetary Geology and Geophysics Grant NNX11AN84G. We thank the IRTF TAC for awarding time to this project, and to the IRTF TOs and MKSS staff for their support. We also thank the anonymous reviewers for their useful comments, which helped to improve the manuscript. 


\section{References}

Asimow, P.D., Ghiorso, M.S., 1998. Algorithmic modifications extending melts to calculate subsolidus phase relation. American Mineralogist 83, 1127-1132.

Bell, J.F., Davis, D.R., Hartmann, W.K., Gaffey, M.J., 1989. Asteroids - The big picture, in: Binzel, R.P., Gehrels, T., Matthews, M.S. (Eds.), Asteroids II, pp. 921-945.

Binzel, R.P., Birlan, M., Bus, S.J., Harris, A.W., Rivkin, A.S., Fornasier, S., 2004. Spectral observations for near-Earth objects including potential target 4660 Nereus : Results from Meudon remote observations at the NASA Infrared Telescope Facility (IRTF). Planetary and Space Science 52, 291-296.

Bischoff, A., Vogel, N., Roszjar, J., 2011. The Rumuruti chondrite group. Chemie der Erde / Geochemistry 71, 101-133.

Bottke, W.F., Asphaug, E., 2013. On the Origin and Evolution of Differentiated Planetesimals. LPI Contributions 1719, 1672.

Bottke, W.F., Nesvorný, D., Grimm, R.E., Morbidelli, A., O’Brien, D.P., 2006. Iron meteorites as remnants of planetesimals formed in the terrestrial planet region. Nature 439, 821-824.

Brearley, A.J., Jones, R.H., 1998. Chondritic Meteorites, in: Papike, J.J. (Ed.), Reviews in Mineralogy Vol. 36, Planetary Materials. Mineralogical Society of America, pp. 3-1-3-398.

Brunetto, R., de León, J., Licandro, J., 2007. Testing space weathering models on A-type asteroid (1951) Lick. Astronomy and Astrophysics 472, 653-656.

Burbine, T.H., Binzel, R.P., 2002. Small Main-Belt Asteroid Spectroscopic Survey in the NearInfrared. Icarus 159, 468-499.

Burbine, T.H., Buchanan, P.C., Dolkar, T., Binzel, R.P., 2009. Pyroxene mineralogies of nearEarth vestoids. Meteoritics and Planetary Science 44, 1331-1341.

Burbine, T.H., McCoy, T.J., Hinrichs, J.L., Lucey, P.G., 2006. Spectral properties of angrites. Meteoritics and Planetary Science 41, 1139-1145.

Burbine, T.H., Meibom, A., Binzel, R.P., 1996. Mantle material in the main belt: Battered to bits? Meteoritics and Planetary Science 31, 607-620.

Burns, R.G., 1993. Mineralogical Applications of Crystal Field Theory. Cambridge, UK: Cambridge University Press, 575pp.

Bus, S.J., 1999. Compositional structure in the asteroid belt: Results of a spectroscopic survey. Ph.D. thesis. Massachusetts Institute of Technology.

Bus, S.J., Binzel, R.P., 2002a. Phase II of the Small Main-Belt Asteroid Spectroscopic Survey A Feature-Based Taxonomy. Icarus 158, 146-177.

Bus, S.J., Binzel, R.P., 2002b. Phase II of the Small Main-Belt Asteroid Spectroscopic Survey The Observations. Icarus 158, 106-145.

Chapman, C.R., 1986. Implications of the inferred compositions of asteroids for their collisional evolution. Societá Astronomica Italiana, Memorie 57, 103-114. 
Christou, A.A., 2013. Orbital clustering of martian Trojans: An asteroid family in the inner Solar System? Icarus 224, 144-153.

Clark, B.E., Bus, S.J., Rivkin, A.S., McConnochie, T., Sanders, J., Shah, S., Hiroi, T., Shepard, M., 2004. E-type asteroid spectroscopy and compositional modeling. Journal of Geophysical Research 109, 2001.

Cloutis, E.A., Gaffey, M.J., Jackowski, T.L., Reed, K.L., 1986. Calibrations of phase abundance, composition, and particle size distribution for olivine-orthopyroxene mixtures from reflectance spectra. Journal of Geophysical Research 91, 11641-11653.

Cloutis, E.A., Gaffey, M.J., Smith, D.G.W., Lambert, R.S.J., 1990. Metal Silicate Mixtures: Spectral Properties and Applications to Asteroid Taxonomy. Journal of Geophysical Research 95, 8323-8338.

Cloutis, E.A., Hudon, P., Romanek, C.S., Bishop, J.L., Reddy, V., Gaffey, M.J., Hardersen, P.S., 2010. Spectral reflectance properties of ureilites. Meteoritics and Planetary Science 45, $1668-1694$.

Cruikshank, D.P., Hartmann, W.K., 1984. The meteorite-asteroid connection - Two olivine-rich asteroids. Science 223, 281-283.

Cushing, M.C., Vacca, W.D., Rayner, J.T., 2004. Spextool: A Spectral Extraction Package for SpeX, a 0.8-5.5 Micron Cross-Dispersed Spectrograph. Publications of the Astronomical Society of the Pacific 116, 362-376.

de la Fuente Marcos, C., de la Fuente Marcos, R., 2013. Three new stable L 5 Mars Trojans. Monthly Notices of the Royal Astronomical Society 432, L31-L35.

de León, J., Licandro, J., Serra-Ricart, M., Pinilla-Alonso, N., Campins, H., 2010. Observations, compositional, and physical characterization of near-Earth and Mars-crosser asteroids from a spectroscopic survey. Astronomy and Astrophysics 517, A23, 25 pp.

DeMeo, F.E., Binzel, R.P., Slivan, S.M., Bus, S.J., 2009. An extension of the Bus asteroid taxonomy into the near-infrared. Icarus 202, 160-180.

Dunn, T.L., Burbine, T.H., Bottke, W.F., Clark, J.P., 2013. Mineralogies and source regions of near-Earth asteroids. Icarus 222, 273-282.

Dunn, T.L., McCoy, T.J., Sunshine, J.M., McSween, H.Y., 2010. A coordinated spectral, mineralogical, and compositional study of ordinary chondrites. Icarus 208, 789-797.

Gaffey, M.J., 1976. Spectral reflectance characteristics of the meteorite classes. Journal of Geophysical Research 81, 905-920.

Gaffey, M.J., Burbine, T.H., Piatek, J.L., Reed, K.L., Chaky, D.A., Bell, J.F., Brown, R.H., 1993. Mineralogical variations within the S-type asteroid class. Icarus 106, 573-602.

Ghiorso, M.S., Sack, R.O., 1995. Chemical mass transfer in magmatic processes IV. A revised and internally consistent thermodynamic model for the interpolation and extrapolation of liquid-solid equilibria in magmatic systems at elevated temperatures and pressures. Contributions to Mineralogy and Petrology 119, 197-212. 
Hardersen, P.S., Cloutis, E.A., Reddy, V., Mothé-Diniz, T., Emery, J.P., 2011. The M-/Xasteroid menagerie: Results of an NIR spectral survey of 45 main-belt asteroids. Meteoritics and Planetary Science 46, 1910-1938.

Hinrichs, J.L., Lucey, P.G., 2002. Temperature-Dependent Near-Infrared Spectral Properties of Minerals, Meteorites, and Lunar Soil. Icarus 155, 169-180.

Hiroi, T., Sasaki, S., 2001. Importance of space weathering simulation products in compositional modeling of asteroids: 349 Dembowska and 446 Aeternitas as examples. Meteoritics and Planetary Science 36, 1587-1596.

Kallemeyn, G.W., Rubin, A.E., Wasson, J.T., 1996. The compositional classification of chondrites: VII. The R chondrite group. Geochimica et Cosmochimica Acta 60, 2243-2256.

Keil, K., 1989. Enstatite meteorites and their parent bodies. Meteoritics 24, 195-208.

Keil, K., 2000. Thermal alteration of asteroids: evidence from meteorites. Planetary and Space Science 48, 887-903.

Kelley, M.S., Gaffey, M.J., 2002. High-albedo asteroid 434 Hungaria: Spectrum, composition and genetic connections. Meteoritics and Planetary Science 37, 1815-1827.

King, T.V.V., Ridley, W.I., 1987. Relation of the spectroscopic reflectance of olivine to mineral chemistry and some remote sensing implications. Journal of Geophysical Research 92, 1145711469.

Lazzaro, D., Angeli, C.A., Carvano, J.M., Mothé-Diniz, T., Duffard, R., Florczak, M., 2004. $\mathrm{S}^{3} \mathrm{OS}^{2}$ : the visible spectroscopic survey of 820 asteroids. Icarus 172, 179-220.

Levison, H.F., Bottke, W.F., Gounelle, M., Morbidelli, A., Nesvorný, D., Tsiganis, K., 2009. Contamination of the asteroid belt by primordial trans-Neptunian objects. Nature 460, 364366.

Lim, L.F., Emery, J.P., Mueller, M., Rivkin, A.S., Trilling, D., Burt, B.J., 2011. Olivine Composition of the Mars Trojan 5261 Eureka: Spitzer IRS Data, in: EPSC-DPS Joint Meeting 2011, p. 1199.

Lucas, M.P., Emery, J.P., Takir, D., 2012. Dunites In The Sky? VNIR Spectra Of Six Suspected A-class Asteroids, in: AAS/Division for Planetary Sciences Meeting Abstracts, p. 110.

Masiero, J.R., Mainzer, A.K., Bauer, J.M., Grav, T., Nugent, C.R., Stevenson, R., 2013. Asteroid Family Identification Using the Hierarchical Clustering Method and WISE/NEOWISE Physical Properties. The Astrophysical Journal 770, 7.

Masiero, J.R., Mainzer, A.K., Grav, T., Bauer, J.M., Cutri, R.M., Dailey, J., Eisenhardt, P.R.M., McMillan, R.S., Spahr, T.B., Skrutskie, M.F., Tholen, D., Walker, R.G., Wright, E.L., DeBaun, E., Elsbury, D., Gautier, IV, T., Gomillion, S., Wilkins, A., 2011. Main Belt Asteroids with WISE/NEOWISE. I. Preliminary Albedos and Diameters. The Astrophysical Journal 741, 68 .

McCanta, M.C., Treiman, A.H., Dyar, M.D., Alexander, C.M.O.., Rumble, III, D., Essene, E.J., 2008. The LaPaz Icefield 04840 meteorite: Mineralogy, metamorphism, and origin of an amphibole- and biotite-bearing R chondrite. Geochimica et Cosmochimica Acta 72, 57575780 . 
McCoy, T.J., Dickinson, T.L., Lofgren, G.E., 1999. Partial melting of the Indarch (EH4) Meteorite: A textural, chemical and phase relations view of melting and melt migration. Meteoritics and Planetary Science 34, 735-746.

Mittlefehldt, D.W., Bogard, D.D., Berkley, J.L., Garrison, D.H., 2003. Brachinites: Igneous rocks from a differentiated asteroid. Meteoritics and Planetary Science 38, 1601-1625.

Mittlefehldt, D.W., McCoy, T.J., Goodrich, C.A., Kracher, A., 1998. Non-Chondritic Meteorites from Asteroidal Bodies, in: Papike, J. (Ed.), Reviews in Mineralogy Vol. 36, Planetary Materials. Mineralogical Society of America, pp. 4-001-4-196.

Moroz, L., Schade, U., Wäsch, R., 2000. Reflectance Spectra of Olivine-Orthopyroxene-Bearing Assemblages at Decreased Temperatures: Implications for Remote Sensing of Asteroids. Icarus 147, 79-93.

Mothé-Diniz, T., Carvano, J.M., Bus, S.J., Duffard, R., Burbine, T.H., 2008. Mineralogical analysis of the Eos family from near-infrared spectra. Icarus 195, 277-294.

Nesvorny, D., 2012. Nesvorny HCM Asteroid Families V2.0. NASA Planetary Data System 189.

Pieters, C.M., Hiroi, T., 2004. RELAB (Reflectance Experiment Laboratory): A NASA Multiuser Spectroscopy Facility, in: Mackwell, S., Stansbery, E. (Eds.), Lunar and Planetary Institute Science Conference Abstracts, p. 1720.

Rayner, J.T., Toomey, D.W., Onaka, P.M., Denault, A.J., Stahlberger, W.E., Vacca, W.D., Cushing, M.C., Wang, S., 2003. SpeX: A Medium-Resolution 0.8-5.5 Micron Spectrograph and Imager for the NASA Infrared Telescope Facility. Publications of the Astronomical Society of the Pacific 115, 362-382.

Reddy, V., 2009. Mineralogical Survey of near-Earth asteroid population: Implications for impact hazard assessment and sustainability of life on Earth. Ph.d. dissertation. University of North Dakota. Grand Forks.

Reddy, V., Carvano, J.M., Lazzaro, D., Michtchenko, T.A., Gaffey, M.J., Kelley, M.S., MothéDiniz, T., Alvarez-Candal, A., Moskovitz, N.A., Cloutis, E.A., Ryan, E.L., 2011a. Mineralogical characterization of Baptistina Asteroid Family: Implications for K/T impactor source. Icarus 216, 184-197.

Reddy, V., Gaffey, M.J., Abell, P.A., Hardersen, P.S., 2012a. Constraining albedo, diameter and composition of near-Earth asteroids via near-infrared spectroscopy. Icarus 219, 382-392.

Reddy, V., Nathues, A., Gaffey, M.J., Schaeff, S., 2011b. Mineralogical characterization of potential targets for the ASTEX mission scenario. Planetary and Space Science 59, 772-778.

Reddy, V., Sanchez, J.A., Nathues, A., Moskovitz, N.A., Li, J.Y., Cloutis, E.A., Archer, K., Tucker, R.A., Gaffey, M.J., Paul Mann, J., Sierks, H., Schade, U., 2012b. Photometric, spectral phase and temperature effects on 4 Vesta and HED meteorites: Implications for the Dawn mission. Icarus 217, 153-168.

Rivkin, A.S., Binzel, R.P., Howell, E.S., Bus, S.J., Grier, J.A., 2003. Spectroscopy and photometry of Mars Trojans. Icarus 165, 349-354.

Rivkin, A.S., Trilling, D.E., Thomas, C.A., DeMeo, F., Spahr, T.B., Binzel, R.P., 2007. Composition of the L5 Mars Trojans: Neighbors, not siblings. Icarus 192, 434-441. 
Rubin, A.E., 1997. Mineralogy of meteorite groups. Meteoritics and Planetary Science 32, $231-247$.

Rubin, A.E., Kallemeyn, G.W., 1994. Pecora Escarpment 91002: A member of the new Rumuruti (R) chondrite group. Meteoritics 29, 255-264.

Sanchez, J.A., Michelsen, R., Reddy, V., Nathues, A., 2013. Surface composition and taxonomic classification of a group of near-Earth and Mars-crossing asteroids. Icarus 225, 131-140.

Sanchez, J.A., Reddy, V., Nathues, A., Cloutis, E.A., Mann, P., Hiesinger, H., 2012. Phase reddening on near-Earth asteroids: Implications for mineralogical analysis, space weathering and taxonomic classification. Icarus 220, 36-50.

Schade, U., Wäsch, R., 1999. NIR reflectance spectroscopy of mafic minerals in the temperature range between 80 and $473 \mathrm{~K}$. Advances in Space Research 23, 1253-1256.

Schulze, H., Bischoff, A., Palme, H., Spettel, B., Dreibus, G., Otto, J., 1994. Mineralogy and chemistry of Rumuruti: The first meteorite fall of the new $\mathrm{R}$ chondrite group. Meteoritics $29,275-286$.

Singer, R.B., Roush, T.L., 1985. Effects of temperature on remotely sensed mineral absorption features. Journal of Geophysical Research 901, 12434-12444.

Sunshine, J.M., Bus, S.J., Corrigan, C.M., McCoy, T.J., Burbine, T.H., 2007. Olivine-dominated asteroids and meteorites: Distinguishing nebular and igneous histories. Meteoritics and Planetary Science 42, 155-170.

Sunshine, J.M., Pieters, C.M., 1998. Determining the composition of olivine from reflectance spectroscopy. Journal of Geophysical Research 103, 13675-13688.

Sunshine, J.M., Pieters, C.M., Pratt, S.F., 1990. Deconvolution of mineral absorption bands An improved approach. Journal of Geophysical Research 95, 6955-6966.

Tedesco, E.F., Noah, P.V., Noah, M., Price, S.D., 2004. IRAS Minor Planet Survey V6.0. NASA Planetary Data System 12.

Tholen, D.J., 1984. Asteroid taxonomy from cluster analysis of Photometry. Ph.D. thesis. Arizona Univ., Tucson.

Tholen, D.J., Barucci, M.A., 1989. Asteroid taxonomy, in: Binzel, R.P., Gehrels, T., Matthews, M.S. (Eds.), Asteroids II, pp. 298-315.

Trilling, D.E., Rivkin, A.S., Stansberry, J.A., Spahr, T.B., Crudo, R.A., Davies, J.K., 2007. Albedos and diameters of three Mars Trojan asteroids. Icarus 192, 442-447.

Tsiganis, K., Gomes, R., Morbidelli, A., Levison, H.F., 2005. Origin of the orbital architecture of the giant planets of the Solar System. Nature 435, 459-461.

Veeder, G.J., Matson, D.L., Tedesco, E.F., 1983. The R asteroids reconsidered. Icarus 55, $177-180$.

Walsh, K.J., Morbidelli, A., Raymond, S.N., O’Brien, D.P., Mandell, A.M., 2011. A low mass for Mars from Jupiter's early gas-driven migration. Nature 475, 206-209.

Warner, B.D., Harris, A.W., Vokrouhlický, D., Nesvorný, D., Bottke, W.F., 2009. Analysis of the Hungaria asteroid population. Icarus 204, 172-182. 
Weisberg, M.K., McCoy, T.J., Krot, A.N., 2006. Systematics and Evaluation of Meteorite Classification, in: Lauretta, D. S. \& McSween, H. Y. (Ed.), Meteorites and the Early Solar System II. University of Arizona Press, pp. 19-52.

Xu, S., Binzel, R.P., Burbine, T.H., Bus, S.J., 1995. Small main-belt asteroid spectroscopic survey: Initial results. Icarus 115, 1-35. 